Kai A. Konrad

Florian Morath

\title{
Escalation in Dynamic Conflict: On Beliefs and Selection
}

Max Planck Institute for Tax Law and Public Finance Working Paper 2017 - 05

August 2017

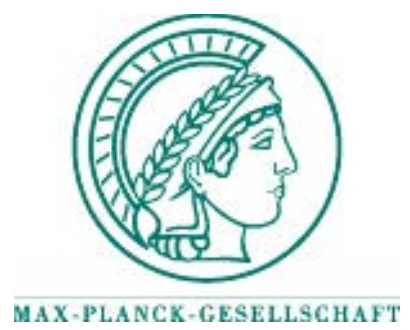

Max Planck Institute for Tax Law and Public Finance

Department of Business and Tax Law Department of Public Economics 
Working papers of the Max Planck Institute for Tax Law and Public Finance Research Paper Series serve to disseminate the research results of work in progress prior to publication to encourage the exchange of ideas and academic debate. Inclusion of a paper in the Research Paper Series does not constitute publication and should not limit publication in any other venue. The preprints published by the Max Planck Institute for Tax Law and Public Finance represent the views of the respective author(s) and not of the Institute as a whole. Copyright remains with the author(s).

Max Planck Institute for Tax Law and Public Finance

Marstallplatz 1

D-80539 Munich

Tel: $\quad+498924246-0$

Fax: $\quad+498924246-501$

E-mail:ssrn@tax.mpg.de

http://www.tax.mpg.de 


\title{
Escalation in dynamic conflict: On beliefs and selection
}

\author{
Kai A. Konrad* Florian Morath ${ }^{\dagger}$
}

August 25, 2017

\begin{abstract}
We study a dynamic multi-stage contest that resolves in each stage only with a given probability. Assuming that there is unobservable heterogeneity in intrinsic motivations we derive properties of the equilibrium efforts across the different stages. Whereas in the corresponding complete information benchmark equilibrium efforts are stable across the stages, uncertainty about the type distribution of possible opponents generates learning. We identify reasons for dynamic adjustments of efforts caused by belief formation and updating and by selection of certain types into continuing conflict. A corresponding experimental setup provides evidence for escalation of efforts in later stages, for type heterogeneity, for belief formation and belief updating, and for selfselection. Overall, our results suggest the importance of an appropriate benchmark model when testing predictions on behavior in conflict or related strategic interactions.

Keywords: Dynamic conflict, lottery contest, heterogeneity, incomplete information, uncertainty, escalation, beliefs, selection, learning, experiment.
\end{abstract}

JEL Codes: C90, D72, D74, D83

*Max Planck Institute for Tax Law and Public Finance, Marstallplatz 1, 80539 Munich. Email: kai.konrad@tax.mpg.de.

$\dagger$ Goethe University Frankfurt, Theodor-W.-Adorno-Platz 4, 60629 Frankfurt am Main, Germany, and Max Planck Institute for Tax Law and Public Finance, Marstallplatz 1, 80539 Munich, Germany. E-mail: morath@econ.uni-frankfurt.de. 


\section{Introduction}

Substantial theoretical and experimental research has been devoted to the study of conflict, described as an adversarial interaction between players who expend efforts and try to achieve mutually exclusive goals. Generic examples include patent races (Loury 1979; Lee and Wilde 1980; Harris and Vickers 1987), procurement (Fullerton and McAfee 1999; Che and Gale 2003), military campaigns, political competition and lobbying (Ellingsen 1991; Baye, Kovenock and de Vries 1993; Klumpp and Polborn 2006; Meirowitz 2008). We study dynamic properties of such conflict if the contestants take part in a sequence of pairwise contests, have incomplete information about their competitor's type and learn from the interaction about the composition of the population from which their future competitors are drawn.

Many of the applications described by models of conflict are dynamic by nature; with incomplete information the dynamics become important even in otherwise stationary environments, as updating of beliefs may cause an escalation or de-escalation of efforts or self-selection (e.g., exit) over the different stages of conflict. For the sake of illustration we may think of lobbying campaigns by interest groups. When players lobby for different policy outcomes, the game may end at a given stage because a final policy decision has been made. However, a new policy-maker or a new government may be elected before the lobbying efforts pay off, making old efforts obsolete and opening up for a new round of efforts. ${ }^{1,2}$

Our framework is based on a dynamic conflict with up to $n$ stages. Each stage takes the form of a two-player Tullock contest in which the prize is awarded with some (exogenous) probability; otherwise the game moves to the next contest stage. In our baseline framework, the players are randomly matched in pairs in each contest stage that they reach so that strategic considerations of how own behavior is interpreted by others are absent. However, if players are uncertain about the composition of the population from which their newly matched rivals are drawn, this may feed back into the dynamics of these encounters via belief formation and updating and possibly via selection whether to continue or to give up.

Next to the theory analysis, we study conflict behavior in a closely corresponding experimental setting. We find evidence for (non-incentivized) heterogeneity among players,

\footnotetext{
${ }^{1}$ Policy reform implementation is an uncertain event. Ben-Bassat's (2011) study highlights the relevance of multiple decision makers in the adoption process of reform and the factors that make adoption more likely. Konrad and Kovenock (2015) allude to the fact that policy makers may default on their promises to interest groups in order to open up for a new lobbying competition and discuss other reasons why policy implementation may fail.

${ }^{2}$ Structurally similar problems emerge in the context of $R \& D$ races: the attempts to innovate may fail in a given period, for exogenous reasons, and for all competitors. Firms may then try a different approach in a new race. Further, in a world with multiple primitive states, repeated violent conflict between them may occur; a given campaign battle may end with the defeat a state and to complete absorption of its resources, but may also fail and lead to a further conflict, possibly against other rival primitive states.
} 
which causes them to update their beliefs about the underlying distribution of types in the population of potential opponents. We identify dynamic effects of contest efforts over time in a multi-stage contest for which the complete information benchmark predicts efforts to be stable. Those adjustment effects are similar in an experimental treatment in which the subjects are constantly re-matched with new opponents and in a treatment in which they repeatedly face the same opponent. Unobservable type heterogeneity and updating about the population of possible opponents also proves to be important for players' self-selection choices in a treatment where the subjects can choose whether to continue or to give up and exit the game. Overall, our experimental results correspond well with the predictions of our theory framework that incorporates incomplete information and population uncertainty. ${ }^{3}$

The analysis sheds light on a methodological issue as well. We study the role of unobservable type heterogeneity in a series of experimental interactions that are strategically linked only via population uncertainty and the possibility to learn about it. With complete information (without heterogeneity among players) the structure would be strategically equivalent to a series of unrelated interactions, but with population heterogeneity and uncertainty about the composition of the population the series of interactions becomes a dynamic Bayesian game. Players may update their beliefs about the population of possible competitors, with implications for self-selection incentives if selection opportunities are available. Unobserved heterogeneity and the implied updating of beliefs is seemingly a driver of the experimental results; not allowing for this type of information asymmetries would lead to theory predictions that are structurally different from the experimental findings.

The role of population uncertainty may be important in various contexts beyond contest applications. Ample evidence has shown that many players who interact in a laboratory environment have motives in addition to the extrinsic monetary incentives provided in the laboratory situation. These include tastes for procedural fairness, for efficiency, for consistent behavior, feelings of altruism or spite, a preference for honesty, a quest for recognition, considerations of self-respect and self-image, status considerations, equity concerns and others. It has been shown that a given population of subjects in the laboratory is heterogenous in the intensity of these motives. ${ }^{4}$ Given that players cannot really know the distribution of types in a subject pool when they enter into an experiment, a well-reasoned choice requires that the player who enters a laboratory session forms a belief about the composition of the

\footnotetext{
${ }^{3}$ The comprehensive survey by Dechenaux, Kovenock and Sheremeta (2015) documents the importance and validity of experimental contest research. Most of the experimental work confronts data with benchmark models that do not account for unobserved intrinsic characteristics of the contestants.

${ }^{4}$ For instance, Kerschbamer, Sutter and Dulleck (2017) develop tests to identify subjects' social preferences (selfish, efficiency loving, spiteful, ineqality averse, inequality loving) and find considerable heterogeneity among subjects.
} 
population of subjects from which possible opponents are drawn, and updates this belief from interaction to interaction.

If players are uncertain about the type composition of the population of possible opponents, it may be appealing early on to make Bayesian inference from one's own type. With an increasing number of observations of others' choices the importance of a player's own type for the beliefs about the opponents' types may fade. The idea that others might be like ourselves and that players give much (actually too much) weight to this hypothesis is studied in social psychology and led to the theory of social projection. In their overview of the 'false consensus effect,' Marks and Miller (1987) survey theories and document empirical evidence on social projection, according to which individuals think that their own choices and judgements are more frequent. Social projection may occur for beliefs, attributes, behaviors, and other personal characteristics, including objectives (see Krispenz, Dickhäuser and Reinhard 2016, p. 867; Kawada et al. 2004). We adopt Bayesian updating about future opponents' subjective valuation of the winner prize to derive theory predictions and to measure their importance in our experiment. Given the substantial amount of findings in the psychology literature that social projection gives too much weight to the own characteristics when making judgments about others, we should not be surprised finding that own behavior has predictive power for expectations about the behavior of others, and that the weight given to one's own type fades too slowly in comparison to proper Bayesian updating.

Our paper is the first to develop and test a theory of sequential contests in which conflict behavior may be driven by intrinsic motives, in which players cannot observe the intensity of the motives of their competitor, and in which they are uncertain about the environment described by the distribution of types of possible competitors. For the laboratory context, but also for many of the major applied problems that are meaningfully described as contests, heterogeneity of competitors, incomplete information about their types, and uncertainty about the contest environment are rather plausible assumptions. Accordingly, both the theoretical predictions and the test of their empirical validity in the laboratory are informative about these problems. We may draw inference from the theory and the experimental outcomes about contestants' learning and updating of beliefs, selection, and the implications of learning and selection for escalation or de-escalation of dynamic conflict. ${ }^{5}$

While the dynamics of conflict caused by population uncertainty and belief updating have not been studied, some elements of this theory relate to results that have been developed in the theory of all-pay contests with incomplete information in a purely static context. Malueg

\footnotetext{
${ }^{5}$ The selection incentives are related to Wärneryd's (2012) analysis of entry in a game in which some players know the value of the prize and others do not. His framework is static and players are ex ante asymmetric with respect to what they know.
} 
and Yates (2004) analyze the static contest between two players whose prize valuations are drawn from a commonly known binary distribution. Even though their information assumption differs from the one that emerges from social projection in our dynamic framework, their results are structurally similar to stage 1 of our game. Fey (2008), Ryvkin (2010), Wasser (2013a, 2013b) and Einy et al. (2015) study existence of Bayesian equilibrium in the static incomplete information Tullock contest. The results by Einy et al. (2015) are closest to our existence results, as they allow players to have private information about the state of nature.

Broad empirical evidence on conflict behavior suggests an intrinsic motivation to win, leading to a mismatch between a complete information benchmark and the experimental results. ${ }^{6}$ Other findings suggest that unobserved heterogeneity of contestants may cause selection effects, or may explain behavior that is difficult to square with the complete-information benchmark model. In line with this, Fu, Gürtler and Münster (2013) show that players sometimes engage in costly messages prior to a lottery contest and explore the role of incomplete information as the rationale for this behavior. Herbst, Konrad and Morath (2015) study the endogenous formation of alliances in contests. They find evidence that players who act under identical monetary incentives differ in their willingness to expend effort, and that players with a high willingness to fight are less inclined to self-select into a fighting alliance with other players. Herbst (2016) finds selection effects which she explains by players' differences in a 'joy of winning.' Our paper points at an implication of intrinsic, non-incentivized type heterogeneity in contest experiments, causing a link between seemingly independent conflict games and turning them into a dynamic Bayesian game. ${ }^{7}$ The experimental results showing a (type-dependent) escalation of efforts across stages correspond well with the theory prediction once type heterogeneity and population uncertainty are taken into account.

\section{Theoretical framework}

\section{$2.1 \quad$ Model}

We consider a framework in which conflict about a prize takes place in up to $n$ stages, each described as a Tullock contest. ${ }^{8}$ The players differ in their prize valuation and there

\footnotetext{
${ }^{6}$ See, for instance, the recent experimental results by Deck and Sheremeta (2012, 2015), Mago, Sheremeta and Yates (2013) and Gelder and Kovenock (2017). Experimental work of Brookins and Ryvkin (2014) compares several types of lottery contests under complete and incomplete information about the effort costs, where the distribution of types is commonly known.

${ }^{7}$ The survey on contest experiments by Sheremeta (2013) discusses various sources for the well-documented heterogeneity in efforts, which are based on unobservable individual characteristics.

${ }^{8}$ Our game can be seen as a Tullock variant of the all-pay-auction game analyzed by Konrad and Kovenock (2015). On the one hand, our game simplifies their framework by assuming that the probability that the game moves to the next stage is strictly exogenous and that the time horizon is finite. On the other hand,
} 
is uncertainty about the probability distribution of types. The theory framework allows for learning about the true type distribution but, by construction, removes strategic aspects of how own effort choices are interpreted by others. One variant of the analysis allows for players' selection.

Players, actions, and timing. Let $I$ be an infinitely large set of players. The game has up to $n$ stages but may end before reaching the terminal stage. In any given non-terminal stage $s<n$, if this stage is reached, each player $i$ is randomly matched with one other player $-i$. Players $i$ and $-i$ simultaneously choose efforts $x_{i, s} \in[\underline{x}, \bar{x}]$ and $x_{-i, s} \in[\underline{x}, \bar{x}]$, where $0<\underline{x}<\bar{x}$. This leads to one of three outcomes, described from the point of view of player $i$. In the first outcome $i$ wins a prize and reaches no further stage (that is, the game ends for $i$ ). In the second outcome player $-i$ wins this prize; again, $i$ reaches no further stage (the game ends for $i$ ). In the third outcome none of the two players wins the prize but the game continues for $i$ who enters stage $s+1$. This third outcome emerges with probability $1-q$. This probability is exogenously given and does not depend on $x_{i, s}$ or $x_{-i, s}$. The other two outcomes emerge with probabilities $q p_{i, s}\left(x_{i, s}, x_{-i, s}\right)$ and $q\left(1-p_{i, s}\left(x_{i, s}, x_{-i, s}\right)\right)$.

The function $p_{i, s}\left(x_{i, s}, x_{-i, s}\right)$ describes the win probability of player $i$ in stage $s$, conditional on the prize being awarded in this stage. This conditional probability is a function of the player's own effort and the opponent $-i$ 's effort at this stage. We assume that this function is given by the Tullock (1980) contest success function:

$$
p_{i, s}\left(x_{i, s}, x_{-i, s}\right)=\frac{x_{i, s}}{x_{i, s}+x_{-i, s}}
$$

for all stages $s=1, \ldots, n .^{9}$ The function $p_{i, s}\left(x_{i, s}, x_{-i, s}\right)$ is continuous, strictly increasing and concave in player $i$ 's own effort and strictly decreasing in the effort of the opponent $-i$ of this stage. ${ }^{10}$

If the prize is not allocated in stage $s<n$ such that player $i$ enters into stage $s+1$, the players are randomly re-matched. Hence, the identity of the opponent typically changes between stages, as the set $I$ of players is infinitely large. We denote by $i$ a given player (with

as the main innovation we allow for incomplete information and learning.

${ }^{9}$ Tullock (1980) introduced (1) in the area of rent-seeking contests. Hirshleifer and Riley (1992), Baye and Hoppe (2003) and Jia (2008) describe microeconomic underpinnings for this function. Skaperdas (1996) and Clark and Riis (1998) offer axiomatic justifications. The function has been used in many areas (see, Konrad 2009, pp. 43-44).

${ }^{10}$ By assuming $x_{i, s} \in[\underline{x}, \bar{x}]$ with $0<\underline{x}$, the denominator in (1) is strictly positive, which avoids the discontinuity problem at $\left(x_{i, s}, x_{-i, s}\right)=(0,0)$ in the standard setup (where $p_{i, s}(0,0)$ needs to be defined separately). The lower bound $\underline{x}$ on efforts should be thought of as being positive but very close to zero. The upper bound $\bar{x}$ should be thought of as being very large such that a choice of $x_{i, s}=\bar{x}$ generates a negative payoff and is dominated by, for instance, $x_{i, s}=\underline{x}$. 
unchanged identity over all stages that are reached) and by $-i$ the opponent assigned to player $i$ in a given stage. In stage $s+1$, player $i$ and the new opponent $-i$ choose efforts $x_{i, s+1} \in[\underline{x}, \bar{x}]$ and $x_{-i, s+1} \in[\underline{x}, \bar{x}]$ and the stage contest resolves according to the same rules as in stage $s$. This continues until the game ends for $i$ because one of the players wins the prize, or until the terminal stage $s=n$ is reached. Interaction at the terminal stage $n$ follows the same rules as in previous stages, with one difference: should none of the two players win at stage $n$, the game ends and no prize is awarded.

Payoffs. Payoffs consist of the prize value if the player wins, minus the own effort costs that are 'all pay': Players $i$ and $-i$ pay the cost of their own efforts $x_{i, s}$ and $x_{-i, s}$. By normalization, these costs are equal to $x_{i, s}$ and $x_{-i, s}$. They occur independent of whether or not a player wins at stage $s$, or whether the prize is awarded in this stage at all. There is no discounting, so effort costs add up for the different stages.

Player $i$ values winning the prize by $v_{i}>0$, which is private information. This fact and the probability model that describes the random process behind the assignment of valuations is common knowledge and formalized below. Player $i$ learns her own prize valuation $v_{i}$ prior to the first effort choice in stage 1 . We assume that a player keeps this valuation of winning throughout all stages of the game. Players may differ in their valuation of the prize: one share of players has a valuation $v_{L}$, the other share of players has a valuation $v_{H}>v_{L} \cdot{ }^{11} \mathrm{We}$ sometimes call a player with valuation $v_{L}$ a 'weak' player and a player with valuation $v_{H}$ a 'strong' player. As there is no discounting, a player has the same benefit from winning the prize if she wins at stage $s$ as if she wins at stage $s+k$.

Altogether, player $i$ 's payoff is $v_{i}-\sum_{k=1}^{k=s} x_{i, k}$ if $i$ wins the prize at stage $s,-\sum_{k=1}^{k=s} x_{i, k}$ if $-i$ wins the prize at stage $s$, and $-\sum_{k=1}^{k=n} x_{i, k}$ if the prize is not allocated at any stage $s=1, \ldots, n$.

Population uncertainty and information structure. The prize valuation $v_{i}$ is assigned to player $i$ in a random process that has two layers of randomness. First, there are two possible states of the world that may prevail. These states $\bar{\omega}$ and $\underline{\omega}$ differ in the probability distribution from which the players' valuations are drawn. All players start with common prior beliefs $\sigma_{i}(\omega)$ about the probability that the world is in state $\omega \in\{\bar{\omega}, \underline{\omega}\}$ and attach a

\footnotetext{
${ }^{11}$ In the experimental framework with monetary prizes, one possible motivation behind the assumption of different prize valuations is that some players may, in addition to the monetary prize, attribute a nonmonetary value to the event of winning, or that they differ in their non-monetary values attributed to winning. We may, for instance, think of $v_{L}$ as the monetary value of the prize and $v_{H}-v_{L}$ as the monetary equivalent of a high type's non-monetary benefit from winning. Such a "joy of winning" has been discussed extensively and analysed experimentally (see Herbst 2016 for a survey of this discussion, and an experiment that focuses on measuring the joy of winning). The difference may also have other reasons, such as differences in altruism or status considerations.
} 
probability of $1 / 2$ to each of the two possible states.

Second, nature draws the type of each player $i \in I$ independently from the same given probability distribution, which depends on the state of the world. Specifically, in state $\omega$ of the world, player $i$ is assigned valuation $v_{H}$ with probability $\pi_{\omega}$, and is assigned valuation $v_{L}$ with the complementary probability $1-\pi_{\omega}$; we assume that

$$
\pi_{\bar{\omega}}=\frac{1}{2}+d \text { and } \pi_{\underline{\omega}}=\frac{1}{2}-d, \text { where } d \in\left(0, \frac{1}{2}\right) .
$$

Hence, the state of the world characterizes the share of high types, $\pi_{\omega}$, and the share of low types, $1-\pi_{\omega}$, in the population; the share of high types is larger in state $\bar{\omega}$ than in state $\underline{\omega}$. For $d>0$ the player's own type as well as the experienced opponents' efforts may have an effect for the players' updating about the probability for the world to be in state $\bar{\omega}$ or $\underline{\omega}$.

Beliefs. At the beginning of stage 1 , each player $i \in I$ learns her valuation $v_{i}$, which $i$ keeps throughout the game. As $v_{i}$ is a random draw from the true probability distribution (which is one of two possible ones), this makes $i$ 's own valuation not only important for the payoff from winning but also for the beliefs about other players' valuations: player $i$ uses Bayes' rule to update her belief about the true state of the world, which is then used to determine her beliefs about the composition of the population from which the opponent $-i$ is drawn. In stages $s \geq 2$ (if reached), the beliefs may also depend on the history of opponents' efforts in previous stages $1, \ldots, s-1$.

Formally, in stage $s$ the population is composed of players with different prize valuations $v_{i}$ and different histories of observed efforts of previous opponents $-i$; the vector

$$
\mathbf{h}_{i, s} \equiv\left(v_{i}, x_{-i, 1}, \ldots, x_{-i, s-1}\right)
$$

describes all relevant information about a player's genuine type (prize valuation) and experience type (history of opponents' effort choices) at the beginning of stage $s$. Somewhat loosely we refer to $\mathbf{h}_{i, s} \in H_{s}$ as $i$ 's 'type' in stage $s$ where $H_{s}$ denotes the set of types in stage $s$. For a player $i$ of type $\mathbf{h}_{i, s}$ to be teamed up with player $j$ of type $\mathbf{h}_{j, s}$, the probability beliefs are characterized by cumulative distribution functions $F_{\mathbf{h}_{i, s}}\left(\mathbf{h}_{j, s}\right)$. Atoms in these distributions will be denoted by $\rho_{\mathbf{h}_{i, s}}\left(\mathbf{h}_{j, s}\right)$; they measure the probability which a player $i$ with valuation $v_{i}$ and experienced opponents' effort $\left(x_{-i, 1}, \ldots, x_{-i, s-1}\right)$ attributes to the event that her newly matched opponent $-i=j$ in stage $s$ has a prize valuation $v_{j}$ and experienced previous opponents with expenditures $\left(x_{-j, 1}, \ldots, x_{-j, s-1}\right)$. 


\subsection{Benchmark: Complete information}

Before analyzing the model with unobserved heterogeneity in the valuation of winning, we characterize the equilibrium of a benchmark case where the incomplete information vanishes, which is the case if $v_{H}=v_{L}=v$.

Proposition 1 Suppose that $v_{H}=v_{L}=v$. Then, a player's equilibrium effort is equal to

$$
\hat{x}_{s}=\frac{q v}{4}
$$

in any stage $s=1, \ldots, n$ if this stage is reached. A player's equilibrium expected continuation payoff at stage $s$ is equal to

$$
\frac{q v}{4} \sum_{k=s}^{k=n}(1-q)^{k-s} .
$$

Proof. The result follows from the standard theory of complete information Tullock contests.

The proposition reveals several interesting properties. First, the resulting equilibrium efforts are the same across all stages. Since the probability $1-q$ that the conflict continues at stage $s$ is independent of the effort choices at stages 1 to $s$, the players choose their effort at a given stage as if their valuation was $q v$, which yields $\hat{x}_{s}=q v / 4$. Second, the players' effort choices in previous stages do not affect the equilibrium effort at a given stage; players' experience does not play any role in this benchmark. Third, the expected continuation payoff and a player's probability to eventually win the prize are decreasing in $s$.

\subsection{Perfect Bayesian Equilibrium}

This section contains the theory results for the framework with incomplete information and uncertainty about the distribution of types. We show existence of a perfect Bayesian equilibrium of the dynamic game and offer a partial characterization of the equilibrium efforts.

Proposition 2 A perfect Bayesian equilibrium in pure strategies exists.

The proofs of this and all subsequent propositions are in the Appendix. In each stage, players form beliefs about the composition of the set of players conditional on their own valuation and the behavior of players they have previously been matched with. The equilibrium beliefs at each stage are characterized by finite sets of mass points $\rho_{\mathbf{h}_{i, s}}\left(\mathbf{h}_{-i, s}\right)$. Based on these the players maximize their expected payoff in the contest of the respective stage. Compactness and continuity properties of the optimal choices allow us to apply Brower's fixed 
point theorem to conclude that this class of problems has a fixed point that characterizes an equilibrium of the static Bayesian game at each state.

The linkage between stages is via belief updating about the composition of the set of possible opponents. Random re-matching of players at each stage and the size of the set of possible opponents become important here, causing that player $i$ 's effort choice at a given stage only affects the future beliefs of a finite number of other players that form a set of zero probability mass. From a single player's perspective this turns the problem into a sequence of structurally independent Bayesian games.

Stage 1 properties. At the beginning of stage 1, the players' beliefs depend on their own valuation only, that is, types $\mathbf{h}_{i, 1} \in\left\{v_{H}, v_{L}\right\}$.

Proposition 3 In stage $s=1$, players with valuation $v_{H}$ and $v_{L}$, respectively, believe that the share of high-valuation players in the population is given by

$$
\rho_{v_{H}}\left(v_{H}\right)=\frac{1}{2}+2 d^{2} \text { and } \rho_{v_{L}}\left(v_{H}\right)=\frac{1}{2}-2 d^{2} .
$$

Using straightforward Bayesian updating, the beliefs in (6) are derived in two steps. First, type $\mathbf{h}_{i, 1}$ updates her beliefs $\sigma_{\mathbf{h}_{i, 1}}(\omega)$ about the probability that the true state of the world is $\omega \in\{\bar{\omega}, \underline{\omega}\}$. The beliefs about the share of high types in (6) follow directly from $\sigma_{\mathbf{h}_{i, 1}}(\omega)$ and Bayes' rule. As (6) shows, each player believes that the state of the world is more likely in which the player's own type is more likely, and thus believes that it is more likely to face an opponent of the same type. Proposition 3 can explain if players of different types form different beliefs about their opponent's type and, hence, effort in the first stage. The next proposition characterizes explicitly the stage 1 equilibrium efforts $x_{\mathbf{h}_{i, 1}}^{*}$ and the players' expectations $E_{\mathbf{h}_{i, 1}}\left(x_{-i, 1}\right)$ about their opponent's effort.

Proposition 4 Denote the equilibrium efforts in stage 1 by $x_{v_{H}}^{*}$ and $x_{v_{L}}^{*}$ for players with valuations $v_{H}$ and $v_{L}$. If $\left(x_{v_{H}}^{*}, x_{v_{L}}^{*}\right) \in(\underline{x}, \bar{x})^{2}$, these efforts are equal to

$$
x_{v_{H}}^{*}=\rho_{v_{H}}\left(v_{H}\right) \frac{q v_{H}}{4}+\left(1-\rho_{v_{H}}\left(v_{H}\right)\right) \frac{q v_{H}^{2} v_{L}}{\left(v_{H}+v_{L}\right)^{2}}
$$

and

$$
x_{v_{L}}^{*}=\rho_{v_{L}}\left(v_{H}\right) \frac{q v_{H} v_{L}^{2}}{\left(v_{H}+v_{L}\right)^{2}}+\left(1-\rho_{v_{L}}\left(v_{H}\right)\right) \frac{q v_{L}}{4} .
$$

Equilibrium beliefs about the opponent's expected effort are

$$
E_{v_{H}}\left(x_{-i, 1}\right)=\rho_{v_{H}}\left(v_{H}\right) x_{v_{H}}^{*}+\left(1-\rho_{v_{H}}\left(v_{H}\right)\right) x_{v_{L}}^{*}
$$


and

$$
E_{v_{L}}\left(x_{-i, 1}\right)=\rho_{v_{L}}\left(v_{H}\right) x_{v_{H}}^{*}+\left(1-\rho_{v_{L}}\left(v_{H}\right)\right) x_{v_{L}}^{*}
$$

with

$$
E_{v_{H}}\left(x_{-i, 1}\right)>E_{v_{L}}\left(x_{-i, 1}\right) .
$$

In the stage 1 contest, the equilibrium effort of a player of type $\mathbf{h}_{i, 1} \in\left\{v_{H}, v_{L}\right\}$ turns out to be a weighted average of the efforts in the corresponding complete information contests in which the valuations of winning are commonly known, that is, for valuations $\left(v_{i}, v_{-i}\right) \in$ $\left\{\left(v_{i}, v_{H}\right),\left(v_{i}, v_{L}\right)\right\}$. In equations $(7)$ and (8), the weights assigned to the two possible effort levels depend on the own type and are equal to player $i$ 's beliefs $\rho_{i}\left(v_{H}\right)$ and $1-\rho_{i}\left(v_{H}\right)$ about the respective population shares. Since $\rho_{v_{H}}\left(v_{H}\right)>\rho_{v_{L}}\left(v_{H}\right)$, strong types place more weight on the possibility that $-i$ is a strong type as well, and vice versa. These different weights generate the two different conjectures (9) and (10) about the expected effort of the opponent. With (11), the expectation of the opponent's effort is positively correlated with the own effort.

It is known that it is difficult to solve analytically for the equilibrium of the Tullock contest with incomplete information. Only partial results exist in the literature. ${ }^{12}$ The equilibrium described in Propositions 3 and 4 considers the case of players who are drawn from the same distribution but can differ in their beliefs about the underlying distribution of types, as a consequence of uncertainty about the true type distribution. A further comparative static result is stated as a corollary. ${ }^{13}$

Corollary 1 The stage 1 equilibrium effort $x_{v_{H}}^{*}$ of strong types is strictly increasing in $\rho_{v_{H}}\left(v_{H}\right)$ and the stage 1 equilibrium effort $x_{v_{L}}^{*}$ of weak types is strictly decreasing in $\rho_{v_{L}}\left(v_{H}\right)$.

This result is in line with standard intuition in contest theory: players exert more effort if they believe it is likely to meet another player with the same (a similar) valuation. Thus, if strong types believe that it is more likely to be in state $\bar{\omega}$ (with many strong types) they adjust their effort upward. If weak types believe that it is more likely to be in state $\bar{\omega}$ they adjust their effort downward. With (6), both types' efforts in stage 1 go up if the distance $d$ between the two possible states of the world is increased. A higher value of $d$ implies that the true type distribution is more asymmetric; hence, stage 1 beliefs react more strongly to the information about the own type and players expect their opponent to be of the same type with higher probability.

\footnotetext{
${ }^{12}$ For instance, Malueg and Yates (2004) offer a solution for the equilibrium with ex ante symmetry of players and homogenous beliefs about the distribution from which the opponent is drawn. Serena (2016) offers some comparative static results, but it becomes clear that analytical characterizations of the equilibrium cannot be obtained in general.

${ }^{13}$ With (7) and (8), Corollary 1 follows from $v_{H}^{2} v_{L}\left(v_{H}+v_{L}\right)^{2}<v_{H} / 4$ and $v_{H} v_{L}^{2} /\left(v_{H}+v_{L}\right)^{2}<v_{L} / 4$.
} 
Later stages. In stages $s \geq 2$, player $i$ 's 'type' is characterized by the own prize valuation and a history of encounters with other players $-i$ with their own valuation $v_{-i}$ and history of previously matched players. If $H_{s}$ contained already $m$ different player types, then any player can be matched with any of these types, such that the set $H_{s+1}$ contains $m^{2}$ elements. Thus, an equilibrium characterization for later stages becomes a challenging issue. In Appendix A.4 we consider properties of stage $s=2$ and establish a ranking of equilibrium efforts (Proposition 7) that demonstrates the potentially countervailing effects of valuation type and experience type on incentives to exert effort. Moreover, we show that the stage 2 equilibrium beliefs about the opponent's effort satisfy

$$
E_{\left(v_{H}, x_{v_{H}}^{*}\right)}\left(x_{-i, 2}\right)>E_{\left(v_{H}, x_{v_{L}}^{*}\right)}\left(x_{-i, 2}\right)=E_{\left(v_{L}, x_{v_{H}}^{*}\right)}\left(x_{-i, 2}\right)>E_{\left(v_{L}, x_{v_{L}}^{*}\right)}\left(x_{-i, 2}\right)
$$

where, in the subscript, the first element refers to the player's own valuation and the second element refers to the effort of the stage 1 opponent. Hence, a player's expectation of the opponent's effort is still correlated with the own valuation type so that high-valuation players expect, on average, higher opponent's effort than low-valuation players. It is evident that calculating the equilibrium efforts for this problem becomes increasingly intractable in later stages. However, we can consider a limit case where the maximum number of stages grows very large and discuss changes in beliefs and efforts across the stages on an intuitive basis.

If the opponents' effort choices remain informative about the type distribution, the impact of the own type on the players' beliefs in stage $s$ becomes less and less important in later stages, as the number of signals obtained increases rapidly (the opponent's effort in stage $s$ is not only informative about this opponent's valuation but also about the opponent's experience in previous stages). In the limit case after a sufficient number of stages, the heterogeneity in beliefs should disappear and all players' beliefs about the share of players with a high prize valuation converge to the true share $\pi_{\omega}$ (where $\omega \in\{\bar{\omega}, \underline{\omega}\}$ ). Moreover, the players anticipate that their opponent will have the same beliefs with probability (close to) one. Therefore, the average equilibrium effort of high-valuation (low-valuation) players converges to the equilibrium effort of high-valuation (low-valuation) players in a contest in which the players have common beliefs about the type distribution. Moreover, the correlation between a player's own effort and the average effort she expects from her stage $s$ opponent identified in (11) and (12) above vanishes in later stages:

$$
\lim _{s \rightarrow \infty} E_{i, s}\left(x_{-i, s} \mid v_{i}=v_{H}\right)=\lim _{s \rightarrow \infty} E_{i, s}\left(x_{-i, s} \mid v_{i}=v_{L}\right)
$$

If the true state is state $\bar{\omega}$ with many strong types, the players' beliefs about the share of strong types are corrected upward in later stages compared to the players' beliefs at stage 1. 
This holds in particular for low-valuation players who initially believe that there are more weak types (compare (6)). The opposite holds in state $\underline{\omega}$. Based on this adjustment in beliefs we can compare the equilibrium efforts in "very late" stages, where the true share of high types is (basically) common knowledge, to the stage 1 equilibrium efforts $x_{v_{H}}^{*}$ and $x_{v_{L}}^{*}$ given in $(7)$ and (8).

Proposition 5 Denote by $\breve{x}_{v_{H}}\left(\pi_{\omega}\right)$ and $\breve{x}_{v_{L}}\left(\pi_{\omega}\right)$ the equilibrium efforts in case there is common knowledge about the true share $\pi_{\omega}$ of high-valuation players and assume that $\left(\breve{x}_{v_{H}}, \breve{x}_{v_{L}}\right) \in$ $(\underline{x}, \bar{x})^{2}$.

(i) If the state of the world is $\omega=\bar{\omega}$ (with many strong types), then $\breve{x}_{v_{L}}<x_{v_{L}}^{*}$.

(ii) If the state of the world is $\omega=\underline{\omega}$ (with many weak types), then $\breve{x}_{v_{L}}>x_{v_{L}}^{*}$ and $\breve{x}_{v_{H}}<x_{v_{H}}^{*}$.

Proposition 5 provides a theoretical foundation for the empirical analysis below. In particular, it makes a prediction on the adjustments of efforts of low and high types in late stages as compared to stage 1 depending on the skewness of the type distribution. More informally, if the distribution of types is right-skewed so that there are more low-valuation players, the average effort of strong types should be decreasing and the average effort of weak types should be increasing in later stages. And if the distribution of types is left-skewed so that there are more high-valuation players, the average effort of strong types should be increasing and the average effort of weak types should be decreasing in later stages. ${ }^{14}$ These different dynamics reflect the intuition that players increase their effort if they learn that it is likely to face an opponent with a similar valuation, and reduce their effort if they learn that the contest is likely to be asymmetric.

An exit option and self-selection. A modified version of the game allows players to exit the game at the end of stage 1 in case the conflict has not yet been resolved. Formally, after observing the outcome of the stage 1 contest, all players simultaneously and independently decide whether to exit the game. In case of exit a player receives a fixed payment $b$ but does no longer participate in stages $2, \ldots, n$. For the players who do not exit the game continues with possible contest stages $s=2, \ldots, n$ within the population of players who did not exit. If all but a set of players with mass zero exit, the game ends for all players.

Proposition 6 Suppose that exit is possible at the end of stage 1. For an exit payment $b \in\left[b_{L}, b_{H}\right]$, there is a perfect Bayesian equilibrium in which all players with valuation $v_{L}$

\footnotetext{
${ }^{14}$ In theory the ranking between effort $\breve{x}_{v_{H}}\left(\pi_{\bar{\omega}}\right)$ and stage 1 effort $x_{v_{H}}^{*}$ is ambiguous since the strong reduction in $\breve{x}_{v_{L}}\left(\pi_{\bar{\omega}}\right)$ as compared to $x_{v_{L}}^{*}$ weakens the high-valuation types' incentive to exert effort. In state $\bar{\omega}, \breve{x}_{v_{H}} \leq x_{v_{H}}^{*}$ occurs, however, only for "extreme" parameter values; for details see the proof of Proposition 5 .
} 
exit and all players with valuation $v_{H}$ do not exit, where $b_{L}$ and $b_{H}$ are given by

$$
b_{L} \equiv\left[q \frac{\max \left\{\tilde{x}_{L}, \underline{x}\right\}}{\max \left\{\tilde{x}_{L}, \underline{x}\right\}+\frac{q v_{H}}{4}} v_{L}-\max \left\{\tilde{x}_{L}, \underline{x}\right\}\right] \sum_{t=2}^{n}(1-q)^{t-2}
$$

with $\tilde{x}_{L} \equiv q\left(-v_{H}+2 \sqrt{v_{L} v_{H}}\right) / 4$ and

$$
b_{H} \equiv \frac{q v_{H}}{4} \sum_{t=2}^{n}(1-q)^{t-2}>b_{L} .
$$

At stages $s=2, \ldots, n$ (if reached), all players believe that their opponent has a valuation $v_{H}$ with probability one and equilibrium efforts are equal to

$$
x_{s}^{*}=\frac{q v_{H}}{4} .
$$

For intermediate values of the exit option, there is an equilibrium in which all weak types exit so that the population of players in stages $s \geq 2$ consists of strong types only. The value $b_{H}$ represents the expected continuation payoff of strong types; the constraint $b \geq b_{L}$ ensures that weak types do not want to deviate from the candidate equilibrium. ${ }^{15}$

In the equilibrium in which weak types exit and strong types remain, average effort in stages $s \geq 2$ is strictly higher than average stage 1 effort, due to two effects. ${ }^{16}$ First, there is the direct self-selection effect that causes the population in stages $s \geq 2$ to be composed only of players who care strongly about winning. Second, since in stages $s \geq 2$ strong types correctly anticipate that their opponent will be a strong type, they further increase their effort as compared to stage 1.

\subsection{Summary of the main predictions}

The theoretical analysis provides the basis of four main testable predictions. First, in the standard complete information benchmark, efforts should be constant across all stages

\footnotetext{
${ }^{15}$ This separating equilibrium with selection described in Proposition 6 need not be unique. There is always a trivial equilibrium in which all players exit because they expect that all other players exit. And there can be equilibria with pooling, depending on the size of the exit payment. For instance, an exit payment $b$ that is very close to $b_{L}$ is also compatible with an equilibrium in which no player exits, due to the complementarity of exit decisions. If many weak types remain active, this makes it more likely that other players are matched with a weak type, which makes it more attractive for other weak players to remain active.

${ }^{16}$ By Corollary 1 , the stage 1 effort of a high type is strictly lower than $q v_{H} / 4$ and approaches $q v_{H} / 4$ if the probability $\rho_{v_{H}}\left(v_{H}\right)$ that a strong type assigns to meeting another strong type approaches one. Similarly, stage 2 effort $x_{\left(v_{H}, x_{v_{H}}^{*}\right)}^{*}$ is strictly lower than $q v_{H} / 4$ so that $x_{s}^{*}$ in (16) is also strictly larger than all types' equilibrium stage 2 effort in the framework without exit option. By an equivalent argument, $x_{s}^{*}$ in (16) is strictly larger than the equilibrium efforts $\breve{x}_{v_{H}}\left(\pi_{\bar{\omega}}\right)$ and $\breve{x}_{v_{H}}\left(\pi_{\omega}\right)$ in case all players know the true share of strong and weak types.
} 
(Proposition 1). Second, if there is unobservable heterogeneity in the (intrinsic) valuation of winning and players are uncertain about the true distribution of these valuations among players in the population, then the individual beliefs about the opponent's effort are positively correlated with the own effort in early stages of the game. The correlation should become weaker in later stages of the game (compare Proposition 3 and Proposition 7 in Appendix A.4 as well as the discussion around (13)). Third, if the true type distribution of subjective valuations of the prize consists of many weak types (strong types), weak types' efforts should go up (go down) and strong types' efforts should go down (go up) in later stages, as compared to stage 1 (Proposition 5). Forth, if exit is possible at the end of stage 1 , weak types should exit and strong types should remain so that average effort in stages $2, \ldots, n$ is strictly higher than average stage 1 effort (Proposition 6 ).

\section{$3 \quad$ Experimental design}

\subsection{Treatments}

The baseline experimental treatment BASE corresponds to the theory framework outlined above. The individuals compete in up to $n=5$ stages about a prize of monetary value $v=450$ by choosing investments $x_{i, s}$ from the set $\{1,2, \ldots, 450\}$ in each stage $s$ that is reached. Together with the choice of the own effort, each individual has to state the effort she expects from her opponent in this stage (as a number between 1 and 450); these stated beliefs $E_{i, s}\left(x_{-i, s}\right)$ are not displayed to other players. In each stage, after the effort choices have been made, the individuals observe the investment of their opponent in this stage and a "lottery wheel" determines whether one of the two players is allocated the prize or whether the game proceeds to the next stage. ${ }^{17}$ The exogenous probability that the prize is allocated in a given stage is $q=1 / 3$. At each stage, the individuals are randomly re-matched in pairs.

Two experimental variations depart from this baseline treatment in two different directions. First, the EXIT treatment adds the possibility of exit. As in the modified theory framework described above, the individuals have the option to exit the game at the end of stage 1, after observing the stage 1 efforts and outcome and in case the prize has yet not been allocated in stage 1.18 Individuals make this choice between "exit" and "remain" simultaneously and independently. Denote the stage 1 pair of players by $(i,-i)$. If both $i$

\footnotetext{
${ }^{17}$ The lottery wheel is a circle area with colored segments that represent the two players' win probabilities $q p_{i, s}$ and $q p_{-i, s}$ as well as a grey segment that corresponds the probability $1-q$ that the prize is not allocated at stage $s$. An arrow that rotates around the circle area determines the outcome of stage $s$.

${ }^{18}$ This option is provided at the end of stage 1 only to facilitate the decision-making for the subjects (who do not have to anticipate future contest outcomes and exit choices) and the idenfication of a treatment effect.
} 
and $-i$ choose to exit then for both individuals the game ends with an exit payment of 60 points each (minus the individual cost of stage 1 effort). If both individuals $i$ and $-i$ choose to remain then both enter into stage 2 (where new pairs of subjects are randomly formed). If one individual chooses to exit and her stage 1 opponent chooses to remain then a coin flip decides on whether both subjects exit or enter into stage $2 .{ }^{19}$ Apart from adding this exit option, the sequence of actions in this treatment is exactly as in the BASE treatment. The payment in case of exit is chosen to be lower than the equilibrium expected continuation payoff in the benchmark case with symmetric players who maximize their monetary payoffs so that in the latter case no player should exit in equilibrium.

Second, in the FIXED treatment, the individuals repeatedly face the same opponent. More precisely, at the beginning of a game, the subjects are randomly matched in pairs and remain in the same pair for the (up to) five stages. With complete information, the benchmark prediction for this treatment with fixed matching within the game remains as in Proposition 1. Incomplete information, however, adds a signaling problem to the game, as a player's effort in early stages may be informative about this player's type. In the contest framework considered, there are incentives both for signaling a low and a high valuation of winning: Intuitively, signaling a low valuation can yield a moderation of efforts and can, thus, be beneficial, whereas signaling a high valuation can discourage the opponent and, thus, be beneficial as well. ${ }^{20}$ On the other hand, even with a finite number of stages, aspects of "cooperation" may be important, which could result in lower efforts. Therefore, the overall effect on efforts is ambiguous. From an experimental point of view, the FIXED treatment only varies the matching protocol to consider the (theoretically much more complex but empirically relevant) case of an identical opponent across the stages of conflict; the data reveal whether this seemingly minor experimental variation causes a difference in behavior, on average and with respect to adjustments of efforts across the stages. ${ }^{21}$

Each subject takes part in only one treatment. In each treatment, the respective multistage contest is played for 15 times. In other words, each subject plays the same game (with

\footnotetext{
${ }^{19}$ This procedure guarantees that there is an even number of subjects in all stages, and is easy to explain to the subjects. It has the advantage that it yields additional experimental variation by extending the sample in later stages to subjects who actually preferred to exit. Moreover, it would also allow to run treatments with exit option and fixed matching across stages.

${ }^{20}$ Updating about the specific opponent's type adds a strategic link between the stages. In this case, the equilibrium is known to be difficult to solve for even under restrictive assumptions. Münster (2009) identifies strategic "sandbagging" in a model with two stages and common beliefs about the type distribution where $v_{i} \in\left\{0, v_{H}\right\}$.

${ }^{21}$ To the best of our knowledge, the only experiment that directly compares the impact of random versus fixed matching on contest efforts is Lugovskyy, Puzzello, and Tucker (2010) in the context of an all-pay auction with complete information in which the equilibrium involves mixed strategies. They find efforts to be (insignificantly) lower with fixed matching. Sheremeta (2013) conducts a meta-study of experiments on lottery contests and finds no significant effect of the matching protocol on average efforts.
} 
up to 5 stages $s$ ) in 15 rounds $r$. In all treatments we apply random re-matching of subjects between different rounds $r$, also in the FIXED treatment.

\subsection{Experimental procedures}

The experiment was conducted at econlab Munich in 12 sessions (4 sessions per treatment with typically 24 subjects per session), involving 276 subjects in total. The subjects were typically students of Munich universities. ${ }^{22}$ At the beginning of each session each subject was shown a video on the computer screen in which the experimental instructions (also distributed as hard copy) were read aloud. Then, the subjects had to answer a few control questions to ensure they understood the rules of the experiment. After the 15 rounds of the main experiment, we conducted an extended post-experimental questionnaire which, apart from socioeconomic information and questions about the experiment, elicited measures for risk preferences, distributional preferences, ambiguity aversion, loss aversion, and cognitive reflection. At the end of the experiment, 3 out of the 15 rounds and one of the incentivized post-experimental tasks were randomly selected for payment. The resulting amount of money was added to (or subtracted from) an endowment of 10 Euros. On average a session lasted 90 minutes and the average payment was 17.80 Euros plus a show-up fee of 6 Euros.

For the experimental sessions, the randomization of whether or not in a given stage $s \in\{1, \ldots, 5\}$ of round $r \in\{1, \ldots, 15\}$ the prize would be allocated (with the exogenous probability $q=1 / 3$ ) was conducted (but not announced) before the start of the first session and was kept the same across all treatments, sessions, and subject pairs. ${ }^{23}$ In other words, the number of stages to be played within round $r \in\{1, \ldots, 15\}$ was the same for all subjects. This ensures that learning about the game and the numbers of signals obtained about the distribution of types are identical across treatments and sessions at any stage $s$ of round $r$. The random re-matching took place in subgroups (typically 8 subjects, although the precise size of the matching groups was not made explicit) in order to gain more independence of observations and allow us to investigate learning dynamics across different populations.

\footnotetext{
${ }^{22}$ The subjects were recruited using the software ORSEE (Greiner 2004). About one half of the students were female, $20 \%$ studied economics or a related field, and the average age was 23.0.

${ }^{23}$ Before running the experiment we independently drew $15 \times 5$ random numbers $y_{r s}$ from a uniform distribution on the unit interval so that (i) a number $y_{r s} \leq 1 / 3$ indicated that in stage $s$ of round $r$ (if reached) the prize would be allocated based on the player's effort choices and (ii) a number $y_{r s}>1 / 3$ indicated that in stage $s$ of round $r$ (if reached) the prize would not be allocated but the game would proceed to stage $s+1$ (or end if $s=5$ ).
} 

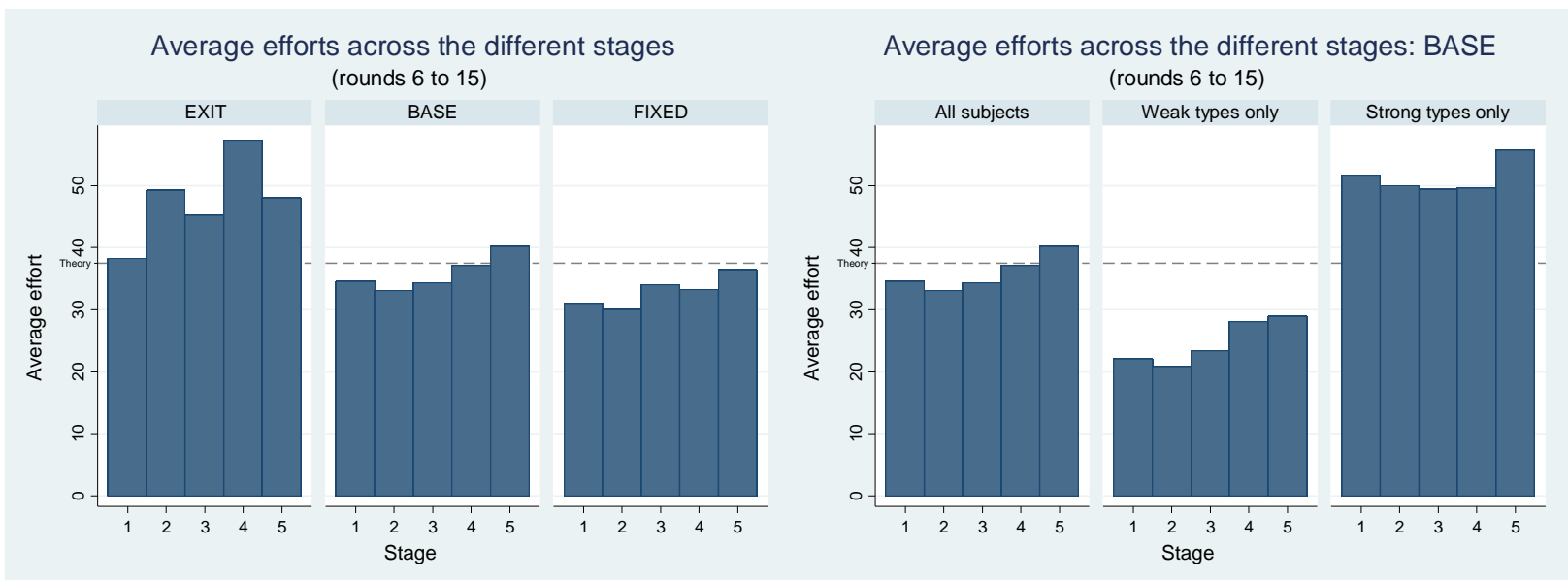

Figure 1: Average effort choices in stages 1 to 5, by treatment (left panel) and by player type (right panel).

\section{Results}

\subsection{Overview of the main results}

Do the individuals adjust their effort in later stages of the game, and if yes, is there a tendency to escalate or to de-escalate? Figure 1 provides a first answer to this question by plotting average efforts in the five stages. In the treatments BASE and FIXED there is an upward trend in the average efforts, which appears to be similar in both of these treatments even though overall the average efforts are slightly lower with fixed matching within one round than in the baseline treatment with random matching (see the left panel of Figure 1).

In the EXIT treatment, stage 1 efforts are comparable to the two other treatments, but from stage 2 onward after exit was possible there is an upward jump in average efforts (in line with Proposition 6). The higher variance in efforts in the EXIT treatment may be caused by the lower number of observations in stages 2 to 5 (due to exit of a substantial share of individuals; see Section 4.3).

Splitting the subjects of the BASE treatment in subsamples of 'strong' and 'weak' types hints at an asymmetry in how different types adjust their efforts; the right panel of Figure 1 suggests that the escalation observed is mainly driven by players classified as 'weak' types.

\subsection{On individual efforts}

To address the question of whether average efforts change significantly over the different stages we estimate a set of random-effects regressions. The dependent variable is the effort $x_{i r s}$ of individual $i$ in stage $s$ of round $r$. A first set of estimations presented in Table B.1 
in Appendix B.2 checks whether there is a significant increase in average efforts across the stages, as indicated by the left panel of Figure 1. The second set of estimations takes up on the potentially different dynamics for strong and weak types as predicted by the theory and suggested by the right panel of Figure 1. For simplicity we estimate individual effort as a linear function of the stage $s$. The variable included is $s-1$ so that the constant measures the average effort in stage 1 and the coefficient of "Stage ${ }_{s-1}$ " measures the average per-stage change in effort. Our main variables of interest are the variable "Stage ${ }_{s-1}$ " and, in EXIT, an indicator variable $1_{s \geq 2}$ for the observations from stage $s \geq 2$ in order to allow for an effect of the exit option. The main estimations focus on behavior from rounds 6 to 15 where subjects have gained some experience with the multi-stage setup, but we will also point to effects of learning in early rounds.

Escalation on average. Table B.1 in the Appendix contains separate estimations for the three treatments BASE, FIXED, and EXIT as well as an estimation that pools all treatments using treatment dummies and interaction terms with the main explanatory variables "Stage $s_{s-1}$ " and $\mathbf{1}_{s \geq 2}$. All estimations control for the stated beliefs $E_{\text {irs }}\left(x_{-i r s}\right)$ and $\left(E_{\text {irs }}\left(x_{-i r s}\right)\right)^{2}$ (to capture non-monotonicity of the best reply functions) and the opponent's effort $x_{-i r 1}$ in stage 1 of round $r$. Moreover, we include dummy variables for the rounds $r$ and individual-specific control variables obtained from the post-experimental questionnaire.

The main results in Table B.1 show that in BASE and FIXED there is a small but significant upward trend in average efforts of about 1 point per stage (see the coefficient of "Stage ${ }_{s-1}$ " in estimations 1 and 2; $p$-values are 0.034 in BASE and 0.014 in FIXED). In EXIT, average effort in stage 2 increases by 6.1 points as compared to average stage 1 effort (see the coefficient of $1_{s \geq 2}$ in estimation $3 ; p$-value is 0.029 ) but from stage 2 onward there is no further trend in average efforts. ${ }^{24}$ Pooling all data, estimation 4 of Table B.1 shows that the upward trend in efforts across stages is not significantly different in BASE and FIXED (see the coefficient of FIXED $\times$ "Stage ${ }_{s-1} " ; p$-value is 0.430 ).

In all treatments, individual effort is a concave function of the stated beliefs about the opponent's effort. Beyond this effect, the observed effort of the stage 1 opponent has only a significantly positive effect on the own effort in FIXED, as we would expect since in the other treatments the identity of the opponent changes after stage 1 of the game. Among the individual-specific controls, a higher stated willingness to take risks is associated with signif-

\footnotetext{
${ }^{24}$ The results are qualitatively the same when excluding the individual control variables or the round dummies or including dummy variables for the matching groups. This also holds when extending the sample of observations to earlier rounds. Only round 1 turns out to be structurally different, exhibiting a strong downward adjustment of efforts which, in the first contests played, are more than twice the average of later rounds (see Figure B.1 on average efforts across rounds in Appendix B.1).
} 
icantly higher efforts, while a higher CRT score leads to (weakly significantly) lower efforts on average. In the treatment with fixed matching, subjects classified as "altruistic" choose significantly lower efforts, whereas this variable is insignificant in the two other treatments.

Result 1 Average efforts are significantly increasing across stages in all three treatments. The increase is strongest in the EXIT treatment where average efforts go up by $28 \%$ after exit was possible.

Overall we can reject the prediction of the benchmark Proposition 1 under which efforts should be the same across stages. Instead, there is a rather consistent pattern of escalation of efforts in later stages as compared to stage 1, with the strongest effect emerging if selection is possible as in the treatment with exit option (in line with Proposition 6). The treatments with random and with fixed matching are not significantly different in terms of average efforts or average adjustment of efforts.

Escalation by player type. Whereas the theory above makes no definite statement about average adjustments of efforts, it predicts differential effects for different 'types' of players with different intrinsic motivations: players classified as 'weak' and players classified as 'strong.' To allow for such heterogeneity we use an individual's effort choice as a proxy for her valuation of winning and we separate the individuals according to whether their average effort in rounds 1 to 5 is below or above the treatment average in those rounds. ${ }^{25}$ With this classification as weak or strong type, we re-estimate the change in efforts across stages based on the data of rounds 6 to 15, interacting the main explanatory variables "Stage ${ }_{s-1}$ " and $\mathbf{1}_{s \geq 2}$, respectively, with the proxy for a player's type (an indicator variable $\mathbf{1}_{\text {strong type }}$ ).

The estimation results are presented in Table 1. To simplify the exposition we present three separate estimations for the three treatments and focus on a linear trend in efforts (variable "Stage ${ }_{s-1}$ ") in treatments BASE and FIXED and a discontinuity in efforts in stage 2 (indicator variable $\mathbf{1}_{s \geq 2}$ ) in treatment EXIT (in line with the results of estimation 4 of Table B.1). ${ }^{26}$ The large and significant coefficient of the indicator variable $\mathbf{1}_{\text {strong type }}$ in all estimations (treatments) shows that those subjects classified as strong types by their effort in early rounds also choose higher effort in later rounds, compared to weak types. More interestingly, in the treatments without exit option (estimations 1 and 2 of Table 1), the

\footnotetext{
${ }^{25}$ Using the median effort choice as the threshold yields qualitatively very similar results.

${ }^{26}$ The results continue to hold when excluding the individual control variables or the round dummies or including dummy variables for the matching groups. They are also robust to extending the sample of observations to earlier rounds, again with the exception of round 1. Finally, the main results turn out to be even slightly stronger when using the median effort in rounds 1 to 5 of a treatment as the threshold for the type classification as strong or weak.
} 


\begin{tabular}{|c|c|c|c|}
\hline \multicolumn{4}{|c|}{ Dependent variable: individual effort $x_{i r s}$} \\
\hline Indep. var. & $\begin{array}{l}\text { BASE } \\
\text { xtreg } 1\end{array}$ & $\begin{array}{l}\text { FIXED } \\
\text { xtreg } 2\end{array}$ & $\begin{array}{c}\text { EXIT } \\
\text { xtreg } 3\end{array}$ \\
\hline Constant & $\begin{array}{c}22.44^{* * *} \\
(4.898)\end{array}$ & $\begin{array}{c}19.59^{* * *} \\
(3.003)\end{array}$ & $\begin{array}{l}33.23^{* *} \\
(13.986)\end{array}$ \\
\hline $\mathbf{1}_{\text {strong type }}$ & $\begin{array}{c}29.26^{* * *} \\
(5.853)\end{array}$ & $\begin{array}{c}24.00^{* * * *} \\
(3.564)\end{array}$ & $\begin{array}{l}31.91^{* *} \\
(12.436)\end{array}$ \\
\hline Stage $_{s-1}$ & $\begin{array}{c}1.673^{* * *} \\
(0.607)\end{array}$ & $\begin{array}{c}2.411^{* * *} \\
(0.496)\end{array}$ & \\
\hline $\mathbf{1}_{\text {strong type }} \times$ Stage $_{s-1}$ & $\begin{array}{c}-1.516^{*} \\
(0.850)\end{array}$ & $\begin{array}{c}-3.722^{* * *} \\
(0.730)\end{array}$ & \\
\hline $\mathbf{1}_{s \geq 2}$ & & & $\begin{array}{c}4.669 * * \\
(2.122)\end{array}$ \\
\hline $\mathbf{1}_{\text {strong type }} \times \mathbf{1}_{s \geq 2}$ & & & $\begin{array}{l}1.898 \\
(3.346)\end{array}$ \\
\hline Beliefs $E_{\text {irs }}\left(x_{-i r s}\right)$ & $\begin{array}{c}0.316^{* * *} \\
(0.027)\end{array}$ & $\begin{array}{c}0.314^{* * *} \\
(0.023)\end{array}$ & $\begin{array}{c}0.283^{* * *} \\
(0.037)\end{array}$ \\
\hline Beliefs $\left(E_{i r s}\left(x_{-i r s}\right)\right)^{2}$ & $\begin{array}{c}-0.0012^{* * *} \\
(0.000)\end{array}$ & $\begin{array}{c}-0.0006^{* * *} \\
(0.000)\end{array}$ & $\begin{array}{c}-0.0005^{* * *} \\
(0.000)\end{array}$ \\
\hline$-i$ 's effort $x_{-i r 1}$ & $\begin{array}{l}-0.019 \\
(0.015)\end{array}$ & $\begin{array}{c}0.032^{* *} \\
(0.013)\end{array}$ & $\begin{array}{c}-0.022 \\
(0.015)\end{array}$ \\
\hline Round dummies & YES & YES & YES \\
\hline Socioeconomics & YES & YES & YES \\
\hline Observations & 2552 & 2784 & 1760 \\
\hline
\end{tabular}

Note: Random-effects regressions; data from rounds 6 to 15 only; standard errors in parentheses; *** $(* *, *)$ significant at $1 \%(5 \%, 10 \%)$. $\mathbf{1}_{\text {strong type }}=1$ if subject $i$ 's average effort in rounds 1 to 5 higher than average effort of all subjects in rounds 1 to 5 of the respective treatment, and $\mathbf{1}_{\text {strong type }}=0$ otherwise. $\mathbf{1}_{s \geq 2}=1$ if stage $\geq 2$, and $\mathbf{1}_{s \geq 2}=0$ otherwise.

Table 1: Individual effort over stages 1 to 5: Strong vs. weak types. 
highly significant effect of the variable "Stage ${ }_{s-1}$ " now measures an increase of efforts across stages by weak types. For those types, the estimated average escalation of efforts per stage is 1.67 in BASE and 2.41 in FIXED. Moreover, the adjustment of efforts is significantly different for strong and weak types, as indicated by the coefficient of the interaction term $\mathbf{1}_{\text {strong type }} \times$ "Stage $_{s-1}$ " in estimations 1 and 2. For the strong types, efforts do not change across the stages in BASE, and decrease across the stages in FIXED. ${ }^{27}$ Hence, the observed increase in efforts in the treatments without exit option is driven by the weak types. In the EXIT treatment, both weak types' effort and strong types' effort significantly goes up from stage 2 onward (by 4.67 and 6.57 points, respectively), but the increase in efforts is not statistically different for weak and strong types. ${ }^{28}$

Result 2 In the treatments without exit option, the increase in efforts in later stages is caused by weak types. For strong types we find no such increase in efforts.

How do the results on efforts presented so far relate to the theory of updating of beliefs under uncertainty about the type distribution? According to the conjecture based on Proposition 5, the weak types' average effort is increasing and the strong types' average effort is decreasing across stages if the true state of the world is state $\underline{\omega}$ with many low-valuation types. Using again a subject's average effort in rounds 1 to 5 as a proxy for the subject's valuation type, Figure 2 shows that the empirical type distribution is right-skewed, suggesting that the type-dependent adjustments of efforts observed across stages are in line with what the theory predicts for the underlying type distribution with many weak types (compare Proposition 5(ii)). ${ }^{29}$ That said, we do not want to claim that the theory of updating of beliefs is the sole mechanism that drives the observed results of escalation. In later rounds, the informational value of observing the opponent's effort in a given stage becomes rather small, and the individuals should also update their beliefs across rounds, so that the increase

\footnotetext{
${ }^{27}$ In BASE, the sum of "Stage ${ }_{s-1}$ " and its interaction with the indicator variable $\mathbf{1}_{\text {strong type }}$ is close to zero and insignificant ( $p$-value is 0.821); in FIXED, the sum of "Stage ${ }_{s-1}$ " and its interaction with the indicator variable $\mathbf{1}_{\text {strong type }}$ is negative and significant ( $p$-value is 0.033 ).

${ }^{28}$ In estimation 3 for EXIT, the coefficient of $1_{s \geq 2}$ measures the increase of efforts in stages $s \geq 2$ for weak types ( $p$-value is 0.028 ) and the sum of the coefficients of $1_{s \geq 2}$ and $1_{s \geq 2} \times 1_{\text {strong type }}$ measures the increase of efforts in stages $s \geq 2$ for strong types ( $p$-value is 0.016 ).

${ }^{29}$ Looking at the empirical type distribution within a given population (matching group), we can also re-run the estimations of Table 1 by including a three-way interaction term of $\mathbf{1}_{\text {strong type }}$ "Stage ${ }_{s-1}$ " and a variable indicating the share of players classified as 'strong' within a given matching group. The results show that weak types' increase of efforts across stages becomes larger in matching groups with many weak types but does not occur in matching groups with many strong types. Similarly, strong types' efforts decrease more strongly across stages in matching groups with many weak types but increase across stages in matching groups with many strong types. Although the coefficients on the interaction terms with the indicator for the share of strong types are imprecisely measured, these different dynamics are in line with the predictions of Proposition 5, providing further support for the importance of updating of beliefs about the type distribution.
} 


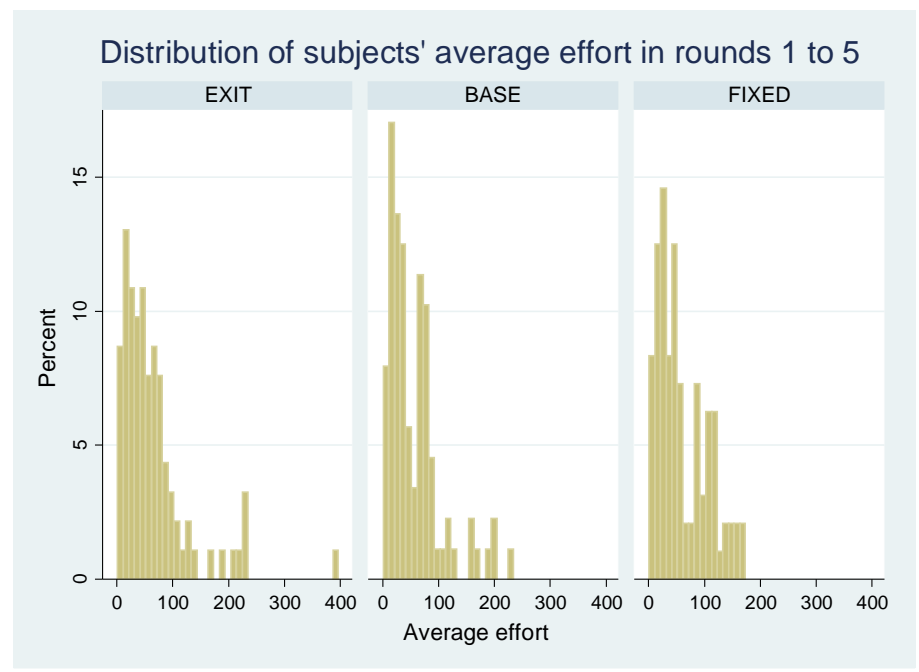

Figure 2: Distribution of 'types' across treatments.

in efforts across stages should disappear in later rounds. ${ }^{30}$ We will come back to the subjects' stated beliefs in Section 4.4. ${ }^{31}$

\subsection{On self-selection}

The estimations on effort choices demonstrated the strongest adjustment effects of effort in the EXIT treatment. To understand the role of selection we run logistic random-effects regressions on individual $i$ 's choice exitir1 whether to exit the game at the end of stage 1 of round $r .^{32}$ The estimation results presented in Table 2 confirm a self-selection effect based on the propensity to invest effort: The probability of a choice of exit is significantly decreasing in the own stage 1 effort; in other words, those subjects with low stage 1 effort are more likely to exit. This biases the sample in stages $s \geq 2$ toward strong types so that equilibrium effort should go up as compared to stage 1 (compare Proposition 6), providing an explanation for

\footnotetext{
${ }^{30}$ On the other hand, in the same way in which subjects' beliefs may place too much weight on the own type, updating of beliefs may occur across stages even in late rounds. If we run the estimations of Table B.1 separately for rounds 6 to 10 and rounds 11 to 15, we find that in the BASE treatment the effect of "Stage ${ }_{s-1}$ " is only significant (and stronger) in rounds 6 to 10 but is smaller and no longer significant in rounds 11 to 15, where updating of beliefs should be of less impact. A corresponding finding is obtained for the type-dependent adjustment of efforts in Table 1. In contrast, the increase in efforts in the EXIT treatment caused by self-selection (measured by $\mathbf{1}_{s \geq 2}$ ) remains robust in rounds 11 to 15 .

${ }^{31} \mathrm{~A}$ complementary explanation of escalation is based on subjective probability weighting in the spirit of Quiggin (1982), Yaari (1987), Prelec (1998) (see the Appendix A.7) An example calculated there for parameter values $v=450$ and $q=1 / 3$ in a given stage and subjective probability weights as suggested in Baharad and Nitzan (2008) yields equilibrium effort choices $\check{x}_{i, 1}=18.33, \check{x}_{i, 2}=18.35, \check{x}_{i, 3}=18.68$, $\check{x}_{i, 4}=19.98$, and $\check{x}_{i, 5}=25.25$. Probability weighting cannot straightforwardly explain differential effects on escalation for subjects with low and high prize valuations, however.

${ }^{32}$ The probability of a choice of "exit" is equal to 0.5 in rounds 1 to 5 and equal to 0.48 in rounds 6 to 15 .
} 


\begin{tabular}{|c|c|c|c|}
\hline \multicolumn{4}{|c|}{ Dependent variable: choice of exit (exit ${ }_{i r 1}=1$ if exit) } \\
\hline Indep. var. & $\begin{array}{c}\text { EXIT } \\
\text { xtlogit } 1\end{array}$ & $\begin{array}{c}\text { EXIT } \\
\text { xtlogit } 2\end{array}$ & $\begin{array}{c}\text { EXIT } \\
\text { xtlogit } 3\end{array}$ \\
\hline Constant & $\begin{array}{c}0.177 \\
(1.018)\end{array}$ & $\begin{array}{c}-0.020 \\
(1.020)\end{array}$ & $\begin{array}{c}-0.230 \\
(1.022)\end{array}$ \\
\hline Own effort $x_{i r 1}$ & $\begin{array}{c}-0.012^{* * *} \\
(0.004)\end{array}$ & $\begin{array}{c}-0.011^{* * *} \\
(0.004)\end{array}$ & $\begin{array}{c}-0.012^{* * *} \\
(0.004)\end{array}$ \\
\hline$-i$ 's effort $x_{-i r 1}$ & $\begin{array}{c}-0.003 \\
(0.003)\end{array}$ & & \\
\hline $\mathbf{1}_{x_{-i r 1}>E_{i r 1}\left(x_{-i r 1}\right)}$ & & $\begin{array}{l}0.515^{*} \\
(0.297)\end{array}$ & \\
\hline$\left(x_{-i r 1}-E_{i r 1}\left(x_{-i r 1}\right)\right) / x_{-i r 1}$ & & & $\begin{array}{c}0.030 * * \\
(0.012)\end{array}$ \\
\hline Round dummies & YES & YES & YES \\
\hline Socioeconomics & YES & YES & YES \\
\hline Observations & 736 & 736 & 736 \\
\hline
\end{tabular}

Note: Random-effects logistic regressions; data from rounds 6 to 15 only; standard errors in parentheses; $* * *(* *, *)$ significant at $1 \%(5 \%, 10 \%) . \mathbf{1}_{x_{-i r 1}>E_{i r 1}\left(x_{-i r 1}\right)}=1$ if opponent's actual stage 1 effort $>$ stated beliefs about opponent's effort, and $\mathbf{1}_{x_{-i r 1}>E_{i r 1}\left(x_{-i r 1}\right)}=0$ otherwise.

Table 2: Individual choice whether to exit.

the finding in Result 1.

The estimation results in Table 2 also show that the opponent's stage 1 effort has no significant effect on the probability to exit (compare estimation 1), which is plausible given the random re-matching of player pairs in the subsequent stages. The difference between the stated beliefs $E_{i r 1}\left(x_{-i r 1}\right)$ about the opponent's effort and the actually observed effort $x_{-i r 1}$, however, can explain the choice to exit: Those players who underestimated the opponent's effort are more likely to exit. This holds when using an indicator variable for whether actual opponent's effort $x_{-i r 1}$ is higher than stated beliefs $E_{i r 1}\left(x_{-i r 1}\right)$ (estimation 2 of Table $2 ; p$-value is 0.082 ) or when including the (relative) difference of actual effort $x_{-i r 1}$ and stated beliefs (estimation 2 of Table $2 ; p$-value is 0.011). ${ }^{33}$ Even when players are randomly re-matched in later stages, the update in beliefs following the "negative surprise" of unexpectedly high opponent's effort can make individuals revise their expectations about payoffs to be obtained in later stages and, hence, affect their choice of exit. ${ }^{34}$

\footnotetext{
${ }^{33}$ The (absolute) difference $x_{-i r 1}-E_{i r 1}\left(x_{-i r 1}\right)$ turns out to have a non-linear effect so that the likelihood of exit is increasing and concave in this $x_{-i r 1}-E_{i r 1}\left(x_{-i r 1}\right)$. An explanation could be that strong types hold higher beliefs that deviate more strongly (in absolute terms) from actual opponent's effort but are, at the same time, less likely to exit.

${ }^{34}$ The results in Table 2 are robust to excluding the individual control variables or the round dummies,
} 
Result 3 In the EXIT treatment, individuals who choose low stage 1 effort are more likely to exit. Moreover, individuals who are negatively surprised by high opponent's effort in stage 1 are more likely to exit.

To summarize, the increase in efforts in later stages of the EXIT treatment is clearly caused by self-selection of strong types into continuing conflict. In addition, discouraging signals obtained about the type distribution cause an increase in the probability to exit.

\subsection{On beliefs and updating}

The theory framework predicts that individual beliefs about the opponent's effort in early stages/rounds should be positively correlated with the own type (effort), while this correlation is reduced once the individuals have obtained sufficiently many signals about other players' types through the observed effort choices. Figure 3 plots the correlation coefficient of own effort and stated beliefs about the respective opponent's effort over the 15 rounds and the (up to) 5 stages within one round. It shows a rapid reduction in the correlation in early rounds but then a rather stable positive correlation, suggesting that the own type matters for the beliefs about other players' types even in later stages/rounds. While the reduction in correlation is in line with the theory predictions, the persistence of considerable correlation is not explained in our theory. This persistence is, however, well in line with considerable psychological evidence on social projection, the confirmation bias, and in this context on the primacy effect in belief formation (see Marks and Miller 1987 on social projection, and Nickerson 1998 on the confirmation bias).

As further evidence on updating of beliefs, Table B.2 in Appendix B.3 shows that, similar to the correlation coefficient in Figure 3, the deviation of stated beliefs from the actual effort of the opponent decreases in the number of signals obtained about others' efforts (the number of the state contest played) but at a decreasing rate. Put differently, the accuracy of the stated beliefs increases rapidly across the first stages and rounds but the learning effects weaken in later stages where the individuals should already have a rather accurate prior.

Result 4 Individual beliefs about opponents' efforts are positively correlated with the own type. This correlation is reduced in later stages and rounds.

Within one round, different types of players may adjust their beliefs differently. Table B.3 in Appendix B.4 presents random-effects regressions where we estimate the stated beliefs as including matching group dummies, or extending the sample of observations to all rounds 1 to 15 . Among the individual-specific control variables, the variables capturing the number of siblings, risk aversion, loss aversion and impulsiveness as well as the dummy variable for economics students significantly positively affect the likelihood to exit. 


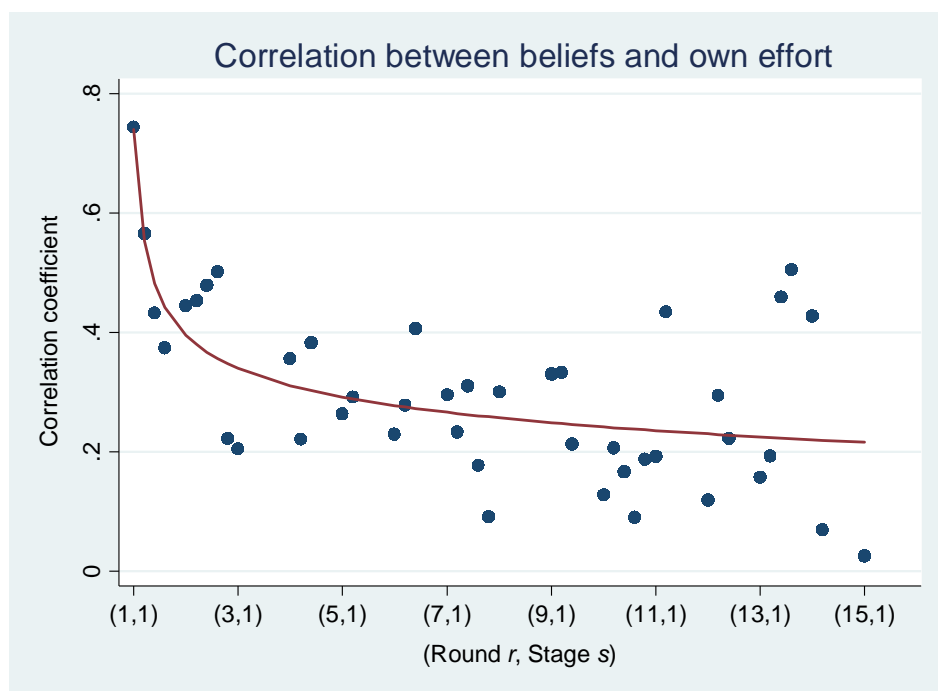

Figure 3: Correlation between own effort $x_{\text {irs }}$ and stated beliefs $E_{\text {irs }}\left(x_{-i r s}\right)$ about the opponent's effort (in a given stage and round).

a function of the stage and distinguish between strong and weak types (we basically use the specifications of Table 1 , replacing the dependent variable by individual beliefs $\left.E_{\text {irs }}\left(x_{-i r s}\right)\right)$. The results confirm the theory predictions in that strong types also hold higher beliefs about the opponent's effort than weak types. Moreover, in BASE, strong types adjust their beliefs downward across stages (the sum of "Stage $s_{s-1}$ " and its interaction with $\mathbf{1}_{\text {strong type }}$ is significant at the $1 \%$ level). Weak types' beliefs, on the other hand, do not significantly adjust across stages. ${ }^{35}$ In FIXED and EXIT, there is no significant adjustment of beliefs over the different stages of a given round. Finally, if we focus on early rounds (rounds 1 to 5) only, the difference in average beliefs between strong and weak types and the updating across stages become stronger (see estimation 4 of Table B.3), again confirming the importance of the own type for belief formation and updating in early stages and rounds.

By and large, we find some evidence for adjustments of beliefs in line with the theory of updating of beliefs under uncertainty about the true type distribution, but the evidence is not unambiguous. We do not want to over-interpret these findings for two reasons. First, the elicitation of beliefs was not incentivized in our experiment and the reliability of measures of beliefs in experimental work is generally debated even when being incentivized. Second, and more specifically to the contest framework, a player's equilibrium effort choice depends on the distribution of types she faces; the average expected effort of the opponent is, therefore, not sufficient to explain individual effort choices, but one would have to elicit the individual

\footnotetext{
${ }^{35}$ With an empirical type distribution exhibiting many weak types, the updating is predicted to be more pronounced for strong than for weak types.
} 
beliefs about the whole distribution of efforts a subject might expect, which arguably leads to a substantial increase of the complexity of the subjects' tasks. With this caveat in mind, our experimental results confirm the importance of beliefs about the opponent's type for individual efforts and the role of the own type for the beliefs about the type distribution.

\section{Conclusions}

This paper studies the importance of heterogeneity in unobserved characteristics and uncertainty about the type distribution for behavior in dynamic contests, focusing on unobserved subjective prize valuations. We derive properties of the equilibrium efforts and investigate individual behavior in a corresponding laboratory experiment. The paper contributes both to the theory of conflict and to the methodology of conflict experiments and related strategic interactions.

From a conflict perspective we study causes and conditions under which efforts in a dynamic conflict may escalate or de-escalate in later stages. We consider a series of Tullock contests in which the prize is allocated only with some probability and the game continues and moves to the next stage (contest) with the remaining probability. Standard theory with symmetric players (complete information) predicts that efforts should be stable across stages in this framework. We develop a theory that allows for heterogeneity of players' (intrinsic) valuations of winning and focuses on incomplete information and uncertainty about the true distribution of these valuations in the population of opponents. Under these plausible conditions players learn from their own type and from the behavior of others and adjust their beliefs and their behavior accordingly. In addition, we identify the effect of players' selection choice between continuation and exit for conflict escalation. Then, we test this theory in a laboratory experiment. We find support for the hypothesis that preference heterogeneity, population uncertainty, and updating of beliefs play a crucial role for conflict escalation or de-escalation.

From a methodological perspective our paper highlights the role of the theoretical benchmark in contest experiments. Systematic deviations from the standard theory benchmark (equilibrium behavior between identical players who maximize their monetary payoffs under conditions of full information) are quite common. But if these systematic deviations are known, the theory benchmark needs to become a different one. It should account for elements such as type heterogeneity in intrinsic (non-incentivized) motivations that are often used to explain the deviations from the standard theory benchmark. The benchmark should adopt these elements. With type heterogeneity and incomplete information, deviations from the standard theory prediction for symmetric players naturally emerge due to differences 
in players' beliefs and experience. And players' experience from previous interactions provides information about the types that players face in later interactions so that adjustments in behavior across interactions are a natural consequence of standard Bayesian updating. From an ex ante perspective (that is, unconditional on the true type distribution), average behavior under the benchmark of symmetric players or complete information may not be qualitatively very different from the average behavior with uncertainty and updating. But ex post (that is, conditional on the true type distribution), this is most likely no longer true. Therefore, the predictions for behavior in a single experiment can be crucially different when taking into account heterogeneity in unobservable characteristics and uncertainty about the true type distribution. Moreover, our experiment suggests that these modified predictions can be a suitable benchmark for testing conflict behavior.

Our experimental results provide evidence for an escalation of efforts even in the absence of players' selection and strategic effects of signaling and signal extraction. The latter are excluded by design of our theory and the experiment, as we randomly re-match the players after each contest stage. Given the simple experimental setup our results appear surprisingly strong. In the experiment, escalation is mainly caused by weak players who increase their effort in later stages of the game (i.e., a given round). The average effects become stronger when allowing for self-selection. Once players are offered an exit option that involves financial rewards, weak players exit the game. Consequently we find an upward jump in effort in line with a straightforward self-selection effect and increased equilibrium efforts of strong players as its strategic consequence. After weak players have left, however, there is no further escalation among strong types.

We also compare the experimental results on escalation of efforts in the absence of signaling effects to a case in which the same pairs of players interact repeatedly and, hence, strategic effects of current effort choices on future outcomes are possible. Contest theory has only derived limited results so far on how signaling incentives in repeated contests affect contest behavior and outcomes. Strategic incentives for information transmission do not play out very strongly in the experiment. We observe very similar results on changes of efforts across stages with fixed as with random matching. The experimental findings on signaling may also interfere with aspects of cooperation in repeated games that emerge in this treatment. Given its empirical relevance, it is interesting to investigate to which degree such strategic effects are able to explain escalation or de-escalation in dynamic conflict; besides pointing at the importance of learning and selection, our results provide a first step into this direction. 


\section{A Proofs of the main theoretical results}

\section{A.1 Proof of Proposition 2}

Suppose that stage $s$ is reached (which implies that the prize has not been awarded in any previous stage) and consider the decision problem of player $i$ who is fully characterized by the vector $\mathbf{h}_{i, s} \equiv\left(v_{i}, x_{-i, 1}, \ldots x_{-i, s-1}\right)$. At stage $s$, a player of type $\mathbf{h}_{i, s}$ forms probability beliefs for all possible types $\mathbf{h}_{j, s} \in H_{s}$. Due to random matching from a set with infinitely many players, these probability beliefs are also the probability beliefs about the type of the opponent $-i$ at stage $s$. The player also forms expectations about each type $\mathbf{h}_{j, s}$ 's effort choice at stage $s$. Assuming that the effort choices are single-valued we denote these by $x_{\mathbf{h}_{j, s}}$.

Denote a player's expected continuation payoff from stage $s+1$ onward by $V_{s+1}\left(\mathbf{h}_{i, s}\right)$. Then, at stage $s$ player $i$ maximizes the objective function

$$
q \int_{\mathbf{h}_{j, s} \in H_{s}} \frac{x_{\mathbf{h}_{i, s}}}{x_{\mathbf{h}_{i, s}}+x_{\mathbf{h}_{j, s}}} v_{i} d F_{\mathbf{h}_{i, s}}\left(\mathbf{h}_{j, s}\right)-x_{\mathbf{h}_{i, s}}+(1-q) V_{s+1}\left(\mathbf{h}_{i, s}\right)
$$

subject to $x \in[\underline{x}, \bar{x}]$. As we will argue further below, the continuation values $V_{s+1+k}\left(\mathbf{h}_{i, s}\right)$ for $k=0, \ldots, n-(s+1)$ are exogenous with respect to a single player's choice at stage $s$. We also will show that, for each stage $s, F_{\mathbf{h}_{i, s}}$ consists of finite sets of probability atoms in the candidate equilibrium. We use these properties to rewrite the objective function as

$$
q \sum_{\mathbf{h}_{j, s} \in H_{s}} \rho_{\mathbf{h}_{i, s}}\left(\mathbf{h}_{j, s}\right) \frac{x_{\mathbf{h}_{i, s}}}{x_{\mathbf{h}_{i, s}}+x_{\mathbf{h}_{j, s}}} v_{i}-x_{\mathbf{h}_{i, s}}+(1-q) V_{s+1}\left(\mathbf{h}_{i, s}\right) .
$$

As $x_{\mathbf{h}_{i, s}} \geq \underline{x}>0$, the objective function is continuous and strictly concave in $x_{\mathbf{h}_{i, s}}$. It must take a unique maximum on the closed and compact support of possible effort choices, and this maximum must be a continuous function of efforts $x_{\mathbf{h}_{j, s}}$. Hence, the optimal choices of the players define a continuous self-mapping

$$
\begin{aligned}
{[\underline{x}, \bar{x}]^{\# H_{s}} } & \rightarrow[\underline{x}, \bar{x}]^{\# H_{s}} \\
\mathbf{x}_{s} & \rightarrow \boldsymbol{\xi}\left(\mathbf{x}_{s}\right)
\end{aligned}
$$

on a convex and compact set, where $\# H_{s}$ is the number of types in stage $s$ emerging from histories in stages $k=1$ to $k=s-1$. Applying Brower's fixed point theorem yields that $\boldsymbol{\xi}\left(\mathbf{x}_{s}\right)$ has at least one fixed point, and this fixed point constitutes a vector of equilibrium efforts at stage $s$. Suppose in what follows that if there are multiple fixed points, the players coordinate on one of them. 
Note that $H_{1}, \ldots, H_{n}$ is comprised of sets with finite numbers of elements on the equilibrium path. For $s=1$, this set has only two elements, as $\mathbf{h}_{i, 1} \in\left\{v_{H}, v_{L}\right\}$. A given set $H_{s}$ with a finite number of types that have positive probabilities that sum up to one leads to a new set of types $H_{s+1}$ in this equilibrium with the same property: the induced set fulfills all assumptions made about $H_{s}$, and in particular, is a finite set if $H_{s}$ was a finite set. So, should the contest not resolve at stage $s$, it moves on to $s+1$ and the problem at $s+1$ has a larger, but finite number of types and is structurally equivalent to the problem at $s$.

Also, a player's belief that $V_{s+1}\left(\mathbf{h}_{i, s}\right)$ is independent of a single player's action at stage $s$ is correct. If a player of type $\mathbf{h}_{j, s}$ deviates from the local equilibrium strategy profile $\mathbf{x}_{s}^{*}$ at stage $s$ and chooses $x \neq x_{\mathbf{h}_{j, s}}^{*}$, the deviating behavior is observed by the stage $s$ opponent $-j$ and changes the history type of this player $-j$. However, given that the contestants are randomly re-matched at each stage and that player $-j$ and all players that $-j$ is matched with constitute a set with zero measure within the set of all players, the deviating behavior does not change the equilibrium composition of types in the next or any other future stage. Therefore, the deviation has no effect on the player's own payoff in future stages.

Hence, the solution for a perfect Bayesian equilibrium of the game reduces to a series of Bayesian equilibria, one for each possible stage of the game. This sequence of problems is linked only by the fact that the local strategy profile at a given stage together with the equilibrium distribution of types $\mathbf{h}_{i, s} \in H_{s}$ at the beginning of stage $s$ jointly determine the equilibrium distribution of types $\mathbf{h}_{i, s+1} \in H_{s+1}$ at the beginning of stage $s+1$.

\section{A.2 Proof of Proposition 3}

Ex ante, all players have the same common prior about the likelihood of the two states of the world. With the common prior belief which assigns a probability of $1 / 2$ to each of the two states, Bayesian updating leads to

$$
\begin{aligned}
\sigma_{v_{H}}(\bar{\omega}) & =\operatorname{prob}\left(\omega=\bar{\omega} \mid v_{i}=v_{H}\right)=\frac{\operatorname{prob}\left(v_{i}=v_{H} \mid \omega=\bar{\omega}\right) \operatorname{prob}(\omega=\bar{\omega})}{\operatorname{prob}\left(v_{i}=v_{H}\right)} \\
& =\frac{\pi_{\bar{\omega}} \frac{1}{2}}{\frac{1}{2} \pi_{\bar{\omega}}+\frac{1}{2} \pi_{\underline{\omega}}}=\frac{1}{2}+d
\end{aligned}
$$

for a player who learns to have a valuation $v_{H}$. This belief induces a belief about the share of strong types in the population and the probability that the stage 1 opponent is of this type. This share/probability is

$$
\rho_{v_{H}}\left(v_{H}\right)=\sigma_{v_{H}}(\bar{\omega}) \pi_{\bar{\omega}}+\left(1-\sigma_{v_{H}}(\bar{\omega})\right) \pi_{\underline{\omega}}=\frac{1}{2}+2 d^{2} .
$$


Analogously, for a player who learns to have a valuation $v_{L}$, Bayesian updating yields

$$
\sigma_{v_{L}}(\bar{\omega})=\operatorname{prob}\left(\omega=\bar{\omega} \mid v_{i}=v_{L}\right)=\frac{\left(1-\pi_{\bar{\omega}}\right) \frac{1}{2}}{\frac{1}{2}\left(1-\pi_{\bar{\omega}}\right)+\frac{1}{2}\left(1-\pi_{\underline{\omega}}\right)}=\frac{1}{2}-d
$$

so that the posterior belief of weak types about the share of strong types is

$$
\rho_{v_{L}}\left(v_{H}\right)=\sigma_{v_{L}}(\bar{\omega}) \pi_{\bar{\omega}}+\left(1-\sigma_{v_{L}}(\bar{\omega})\right) \pi_{\underline{\omega}}=\frac{1}{2}-2 d^{2} .
$$

We note that $\sigma_{v_{H}}(\bar{\omega})>\sigma_{v_{L}}(\bar{\omega})$ and $\rho_{v_{H}}\left(v_{H}\right)>\rho_{v_{L}}\left(v_{H}\right)$.

\section{A.3 Proof of Proposition 4}

The stage 1 effort choice of type $\mathbf{h}_{i, 1} \in\left\{v_{H}, v_{L}\right\}$ maximizes

$$
q\left[\rho_{v_{i}}\left(v_{H}\right) \frac{x_{i, 1}}{x_{i, 1}+x_{v_{H}}^{*}} v_{i}+\left(1-\rho_{v_{i}}\left(v_{H}\right)\right) \frac{x_{i, 1}}{x_{i, 1}+x_{v_{L}}^{*}} v_{i}\right]-x_{i, 1}+V_{2}\left(\mathbf{h}_{i, 1}\right) .
$$

Since the continuation payoff $V_{2}\left(\mathbf{h}_{i, 1}\right)$ does not depend on $x_{i, 1}$, the equilibrium efforts $\left(x_{v_{H}}^{*}, x_{v_{L}}^{*}\right)$ in an interior Bayesian Nash equilibrium are the solution of the system of two equations

$$
\begin{aligned}
& \left(1-\rho_{v_{H}}\left(v_{H}\right)\right) q v_{H} \frac{x_{v_{L}}^{*}}{\left(x_{v_{H}}^{*}+x_{v_{L}}^{*}\right)^{2}}=1-\rho_{v_{H}}\left(v_{H}\right) q v_{H} \frac{1}{4 x_{v_{H}}^{*}} \\
& \rho_{v_{L}}\left(v_{H}\right) q v_{L} \frac{x_{v_{H}}^{*}}{\left(x_{v_{L}}^{*}+x_{v_{H}}^{*}\right)^{2}}=1-\left(1-\rho_{v_{L}}\left(v_{H}\right)\right) q v_{L} \frac{1}{4 x_{v_{L}}^{*}}
\end{aligned}
$$

which are directly obtained from the first-order conditions. Since (21) and (23) imply that $1-\rho_{v_{H}}\left(v_{H}\right)=\rho_{v_{L}}\left(v_{H}\right)$, combining the two equations in (25) yields

$$
\frac{v_{H}}{v_{L}}=\frac{x_{v_{H}}^{*}}{x_{v_{L}}^{*}}
$$

Note that (26) together with $v_{H}>v_{L}$ implies that $x_{v_{H}}^{*}>x_{v_{L}}^{*}$. With (25) and (26) we obtain the equilibrium values (7) and (8). This characterizes the equilibrium effort choices at stage 1 if $\left(x_{v_{H}}^{*}, x_{v_{L}}^{*}\right) \in(\underline{x}, \bar{x})^{2}$. A sufficient condition for the equilibrium to be interior is that $\bar{x} \geq q v_{H}$ and $\underline{x}$ is sufficiently close to zero.

Each player $i$ anticipates the equilibrium effort levels (7) and (8) of their matched opponent $-i$, and these anticipated values are independent of $i$ 's own type. But the players' posterior beliefs about their opponent's type depend on the own type, as given in (21) and (23). Player $i$ uses this posterior belief to calculate the unconditional expected effort of $-i$ as (9) and (10). Due to $\rho_{v_{H}}\left(v_{H}\right)>\rho_{v_{L}}\left(v_{H}\right)$ and $x_{v_{H}}^{*}>x_{v_{L}}^{*}$, we get $E_{v_{H}}\left(x_{-i}\right)>E_{v_{L}}\left(x_{-i}\right)$. 


\section{A.4 Stage 2 properties}

This section provides a partial characterization of the equilibrium in stage 2, which also illustrates why the complexity of the problem increases rapidly if we move from interaction at stage $s=1$ to $s>1$. At stage 2, there are four pairs of player valuation and player experience: $\mathbf{h}_{i, 2} \in H_{2} \equiv\left\{\left(v_{H}, x_{v_{H}}^{*}\right),\left(v_{H}, x_{v_{L}}^{*}\right),\left(v_{L}, x_{v_{H}}^{*}\right),\left(v_{L}, x_{v_{L}}^{*}\right)\right\}$. A player with valuation $v_{i}$ cares not only about her stage 2 opponent's valuation $v_{-i}$ but also about $-i$ 's matching experience, as both affect the opponent's effort choice (the latter through its effect on $-i$ 's beliefs about the composition of the population). Bayesian updating yields the following stage 2 beliefs $\sigma_{\mathbf{h}_{i, 2}}(\omega)$ of types $\mathbf{h}_{i, 2} \in H_{2}$ about the true state of the world: ${ }^{36}$

$$
\sigma_{\left(v_{H}, x_{v_{H}}^{*}\right)}(\bar{\omega})=\frac{1}{2} \frac{(1+2 d)^{2}}{1+4 d^{2}}>\sigma_{\left(v_{H}, x_{v_{L}}^{*}\right)}(\bar{\omega})=\frac{1}{2}=\sigma_{\left(v_{L}, x_{v_{H}}^{*}\right)}(\bar{\omega})>\sigma_{\left(v_{L}, x_{v_{L}}^{*}\right)}(\bar{\omega})=\frac{1}{2} \frac{(1-2 d)^{2}}{1+4 d^{2}} .
$$

Compared to the stage 1 beliefs $\sigma_{\mathbf{h}_{i, 1}}(\omega)$, the stage 2 beliefs of types $\left(v_{H}, x_{v_{H}}^{*}\right)$ (with valuation $v_{H}$ and experience $\left.x_{-i, 1}=x_{H}^{*}\right)$ assign even more probability to the true state of the world being state $\bar{\omega}$ with many strong types (that is, $\sigma_{\left(v_{H}, x_{v_{H}}^{*}\right)}(\bar{\omega})>\sigma_{v_{H}}(\bar{\omega})$ ). The opposite effect emerges for types $\left(v_{L}, x_{v_{L}}^{*}\right)$ where $\sigma_{\left(v_{L}, x_{v_{L}}^{*}\right)}(\bar{\omega})<\sigma_{v_{L}}(\bar{\omega})$. The relation between the own valuation and the players' beliefs is, however, weakened: Types $\left(v_{H}, x_{v_{L}}^{*}\right)$ hold the same beliefs as types $\left(v_{L}, x_{v_{H}}^{*}\right)$. Based on $\sigma_{\mathbf{h}_{i, 2}}(\omega)$ we can directly determine the probability $\rho_{\mathbf{h}_{i, 2}}\left(\mathbf{h}_{-i, 2}\right)$ that type $\mathbf{h}_{i, 2} \in H_{2}$ assigns to her stage 2 opponent being of type $\mathbf{h}_{-i, 2}$, which becomes

$$
\begin{aligned}
\rho_{\mathbf{h}_{i, 2}}\left(\left(v_{H}, x_{v_{H}}^{*}\right)\right) & =\sigma_{\mathbf{h}_{i, 2}}(\bar{\omega}) \pi_{\bar{\omega}}^{2}+\left(1-\sigma_{\mathbf{h}_{i, 2}}(\bar{\omega})\right) \pi_{\underline{\omega}}^{2}, \\
\rho_{\mathbf{h}_{i, 2}}\left(\left(v_{H}, x_{v_{L}}^{*}\right)\right) & =\rho_{\mathbf{h}_{i, 2}}\left(\left(v_{L}, x_{v_{H}}^{*}\right)\right)=\sigma_{\mathbf{h}_{i, 2}}(\bar{\omega}) \pi_{\bar{\omega}}\left(1-\pi_{\bar{\omega}}\right)+\left(1-\sigma_{\mathbf{h}_{i, 2}}(\bar{\omega})\right) \pi_{\underline{\omega}}\left(1-\pi_{\underline{\omega}}\right), \\
\rho_{\mathbf{h}_{i, 2}}\left(\left(v_{L}, x_{v_{L}}^{*}\right)\right) & =\sigma_{\mathbf{h}_{i, 2}}(\bar{\omega})\left(1-\pi_{\bar{\omega}}\right)^{2}+\left(1-\sigma_{\mathbf{h}_{i, 2}}(\bar{\omega})\right)\left(1-\pi_{\underline{\omega}}\right)^{2} .
\end{aligned}
$$

With $\pi_{\bar{\omega}}=1 / 2+d, \pi_{\underline{\omega}}=1 / 2-d$ and $\sigma_{\mathbf{h}_{i, 2}}(\bar{\omega})$ given above, we obtain

$$
\begin{aligned}
\rho_{\left(v_{H}, x_{v_{H}}^{*}\right)}\left(\left(v_{H}, x_{v_{H}}^{*}\right)\right) & =\rho_{\left(v_{L}, x_{v_{L}}^{*}\right)}\left(\left(v_{L}, x_{v_{L}}^{*}\right)\right)=\frac{1}{4}+\frac{5+4 d^{2}}{1+4 d^{2}} d^{2}, \\
\rho_{\left(v_{H}, x_{v_{H}}^{*}\right)}\left(\left(v_{L}, x_{v_{L}}^{*}\right)\right) & =\rho_{\left(v_{L}, x_{v_{L}}^{*}\right)}\left(\left(v_{H}, x_{v_{H}}^{*}\right)\right)=\frac{1}{4}-\frac{3-4 d^{2}}{1+4 d^{2}} d^{2}, \\
\rho_{\mathbf{h}_{i, 2}}\left(\left(v_{H}, x_{v_{H}}^{*}\right)\right) & =\rho_{\mathbf{h}_{i, 2}}\left(\left(v_{L}, x_{v_{L}}^{*}\right)\right)=\frac{1}{4}+d^{2} \text { for } \mathbf{h}_{i, 2} \in\left\{\left(v_{H}, x_{v_{L}}^{*}\right),\left(v_{L}, x_{v_{H}}^{*}\right)\right\}, \\
\rho_{\mathbf{h}_{i, 2}}\left(\left(v_{H}, x_{v_{L}}^{*}\right)\right) & =\rho_{\mathbf{h}_{i, 2}}\left(\left(v_{L}, x_{v_{H}}^{*}\right)\right)=\frac{1}{4}-d^{2} \text { for all } \mathbf{h}_{i, 2} \in H_{2} .
\end{aligned}
$$

\footnotetext{
${ }^{36}$ This result follows similar as in (20) and (22), replacing the prior beliefs $1 / 2$ by $\sigma_{\mathbf{h}_{i, 1}}(\bar{\omega})$.
} 
All types $\mathbf{h}_{i, 2} \in H_{2}$ assign identical probabilities to their opponent being of type $\mathbf{h}_{-i, 2} \in$ $\left\{\left(v_{H}, x_{v_{L}}^{*}\right),\left(v_{L}, x_{v_{H}}^{*}\right)\right\}$. The only difference in beliefs about the type distribution occurs with respect to the shares of types $\left(v_{H}, x_{v_{H}}^{*}\right)$ and $\left(v_{L}, x_{v_{L}}^{*}\right)$.

In the equilibrium of stage 2 , different types hold different beliefs about the type distribution but the players' expectations about each type's equilibrium effort are correct; this equilibrium effort depends on a player's valuation type and experience type. No closed form solution for the stage 2 equilibrium efforts is known to exist. Assuming that the equilibrium is interior, the following partial orderings of the stage 2 equilibrium efforts denoted by $x_{\mathbf{h}_{i, 2}}^{*}$ and the expectations $E_{\mathbf{h}_{i, 2}}\left(x_{-i, 2}\right)$ about the opponent's effort can be established.

Proposition 7 Denote the equilibrium efforts in stage 2 by $x_{\left(v_{i}, x_{v_{j}}^{*}\right)}^{*}$ and assume that $x_{\mathbf{h}_{i, 2}}^{*} \in$ $(\underline{x}, \bar{x})$ for all $\mathbf{h}_{i, 2} \in H_{2}$. Then the following rankings of the stage 2 equilibrium efforts hold:

$$
x_{\left(v_{H}, x_{v_{H}}^{*}\right)}^{*}>x_{\left(v_{H}, x_{v_{L}}^{*}\right)}^{*}>x_{\left(v_{L}, x_{v_{H}}^{*}\right)}^{*} \text { and } x_{\left(v_{H}, x_{v_{H}}^{*}\right)}^{*}>x_{\left(v_{L}, x_{v_{L}}^{*}\right)}^{*}>x_{\left(v_{L}, x_{v_{H}}^{*}\right)}^{*} .
$$

The equilibrium beliefs about the opponent's effort satisfy

$$
E_{\left(v_{H}, x_{v_{H}}^{*}\right)}\left(x_{-i, 2}\right)>E_{\left(v_{H}, x_{v_{L}}^{*}\right)}\left(x_{-i, 2}\right)=E_{\left(v_{L}, x_{v_{H}}^{*}\right)}\left(x_{-i, 2}\right)>E_{\left(v_{L}, x_{v_{L}}^{*}\right)}\left(x_{-i, 2}\right) .
$$

Proof. In an interior equilibrium, the equilibrium efforts satisfy the first-order conditions

$$
\sum_{\mathbf{h}_{-i, 2} \in H_{2}} q \rho_{\mathbf{h}_{i, 2}}\left(\mathbf{h}_{-i, 2}\right) \frac{x_{\mathbf{h}_{-i, 2}}^{*}}{\left(x_{\mathbf{h}_{i, 2}}^{*}+x_{\mathbf{h}_{-i, 2}}^{*}\right)^{2}} v_{i}-1=0, \quad \mathbf{h}_{i, 2} \in H_{2} .
$$

Step 1: $x_{\left(v_{H}, x_{v_{H}}^{*}\right)}^{*}>x_{\left(v_{L}, x_{v_{L}}^{*}\right)}^{*}$. Using (32) for type $\left(v_{H}, x_{v_{H}}^{*}\right)$ and the beliefs in (29),

$$
\begin{gathered}
q \rho_{\left(v_{L}, x_{v_{L}}^{*}\right)}\left(\left(v_{L}, x_{v_{L}}^{*}\right)\right) \frac{1}{4 x_{\left(v_{H}, x_{v_{H}}^{*}\right)}^{*}} v_{H}+q \rho_{\left(v_{L}, x_{v_{L}}^{*}\right)}\left(\left(v_{H}, x_{v_{H}}^{*}\right)\right) \frac{x_{\left(v_{L}, x_{v_{L}}^{*}\right)}^{*}}{\left(x_{\left(v_{H}, x_{v_{H}}^{*}\right)}^{*}+x_{\left(v_{L}, x_{v_{L}}^{*}\right)}^{*}\right)_{H}} \\
+\sum_{\mathbf{h}_{-i, 2} \in\left\{\left(v_{H}, x_{v_{L}}^{*}\right),\left(v_{L}, x_{v_{H}}^{*}\right)\right\}} q \rho_{\left(v_{L}, x_{v_{L}}^{*}\right)}\left(\mathbf{h}_{-i, 2}\right) \frac{x_{\mathbf{h}_{-i, 2}}^{*}}{\left(x_{\left(v_{H}, x_{v_{H}}^{*}\right)}^{*}+x_{\mathbf{h}_{-i, 2}}^{*}\right)^{2}} v_{H}-1=0 .
\end{gathered}
$$

Suppose that $x_{\left(v_{H}, x_{v_{H}}^{*}\right)}^{*} \leq x_{\left(v_{L}, x_{v_{L}}^{*}\right)}^{*}$. Then, together with $v_{L}<v_{H}$, the left-hand side in (33) 
is strictly larger than

$$
\begin{aligned}
q \rho_{\left(v_{L}, x_{v_{L}}^{*}\right)}\left(\left(v_{L}, x_{v_{L}}^{*}\right)\right) & \frac{1}{4 x_{\left(v_{L}, x_{v_{L}}^{*}\right)}^{*}} v_{L}+q \rho_{\left(v_{L}, x_{v_{L}}^{*}\right)}\left(\left(v_{H}, x_{v_{H}}^{*}\right)\right) \frac{x_{\left(v_{H}, x_{v_{H}}^{*}\right)}^{*}}{\left(x_{\left(v_{H}, x_{v_{H}}^{*}\right)}^{*}+x_{\left(v_{L}, x_{v_{L}}^{*}\right)}^{*}\right)_{L}} \\
& +\sum_{\mathbf{h}_{-i, 2} \in\left\{\left(v_{H}, x_{v_{L}}^{*}\right),\left(v_{L}, x_{v_{H}}^{*}\right)\right\}} q \rho_{\left(v_{L}, x_{v_{L}}^{*}\right)}\left(\mathbf{h}_{-i, 2}\right) \frac{x_{\mathbf{h}_{-i, 2}}^{*}}{\left(x_{\left(v_{L}, x_{v_{L}}^{*}\right)}^{*}+x_{\mathbf{h}_{-i, 2}}^{*}\right)^{2}} v_{L}-1,
\end{aligned}
$$

which must be zero by optimality of $x_{\left(v_{L}, x_{v_{L}}^{*}\right)}^{*}$. This establishes a contradiction.

Step 2: $x_{\left(v_{H}, x_{v_{H}}^{*}\right)}^{*}>x_{\left(v_{H}, x_{v_{L}}^{*}\right)}^{*}$. For the beliefs of types $\left(v_{H}, x_{v_{H}}^{*}\right)$ and $\left(v_{H}, x_{v_{L}}^{*}\right)$ in $(29)$, note that $\rho_{\left(v_{H}, x_{v_{H}}^{*}\right)}\left(\left(v_{H}, x_{v_{H}}^{*}\right)\right)-\rho_{\left(v_{H}, x_{v_{L}}^{*}\right)}\left(\left(v_{H}, x_{v_{H}}^{*}\right)\right)=\rho_{\left(v_{H}, x_{v_{L}}^{*}\right)}\left(\left(v_{L}, x_{v_{L}}^{*}\right)\right)-\rho_{\left(v_{H}, x_{v_{H}}^{*}\right)}\left(\left(v_{L}, x_{v_{L}}^{*}\right)\right)$. Defining this difference by $\Delta$, the first-order condition for type $\left(v_{H}, x_{v_{H}}^{*}\right)$ is equivalent to

$$
\begin{aligned}
\sum_{\mathbf{h}_{-i, 2} \in H_{2}} q \rho_{\left(v_{H}, x_{v_{L}}^{*}\right)} & \left(\mathbf{h}_{-i, 2}\right) \frac{x_{\mathbf{h}_{-i, 2}}^{*}}{\left(x_{\left(v_{H}, x_{v_{H}}^{*}\right)}^{*}+x_{\mathbf{h}_{-i, 2}}^{*}\right)^{2}} v_{H}-1 \\
& +q \Delta \frac{x_{\left(v_{H}, x_{v_{H}}^{*}\right)}^{*}}{\left(x_{\left(v_{H}, x_{v_{H}}^{*}\right)}^{*}+x_{\left(v_{H}, x_{v_{H}}^{*}\right)}^{*}\right)^{2}} v_{H}-q \Delta \frac{x_{\left(v_{L}, x_{v_{L}}^{*}\right)}^{*}}{\left(x_{\left(v_{H}, x_{v_{H}}^{*}\right)}^{*}+x_{\left(v_{L}, x_{v_{L}}^{*}\right)}^{*}\right)^{2}} v_{H}=0 .
\end{aligned}
$$

Suppose that $x_{\left(v_{H}, x_{v_{H}}^{*}\right)}^{*} \leq x_{\left(v_{H}, x_{v_{L}}^{*}\right)}^{*}$. Then, using this inequality in the first term in (34) and combining the last two terms, the left-hand side in (34) is (weakly) larger than

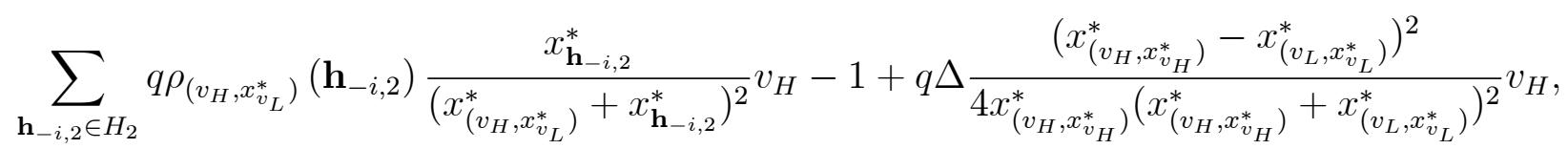

which is strictly larger than zero (using optimality of $\left.x_{\left(v_{H}, x_{v_{L}}^{*}\right)}\right)$. This yields a contradiction.

Step 3: $x_{\left(v_{L}, x_{v_{L}}^{*}\right)}^{*}>x_{\left(v_{L}, x_{v_{H}}^{*}\right)}^{*}$. Using the beliefs of types $\left(v_{L}, x_{v_{L}}^{*}\right)$ and $\left(v_{L}, x_{v_{H}}^{*}\right)$ in $(29)$, we get $\rho_{\left(v_{L}, x_{v_{L}}^{*}\right)}\left(\left(v_{L}, x_{v_{L}}^{*}\right)\right)-\rho_{\left(v_{L}, x_{v_{H}}^{*}\right)}\left(\left(v_{L}, x_{v_{L}}^{*}\right)\right)=\rho_{\left(v_{L}, x_{v_{H}}^{*}\right)}\left(\left(v_{H}, x_{v_{H}}^{*}\right)\right)-\rho_{\left(v_{L}, x_{v_{L}}^{*}\right)}\left(\left(v_{H}, x_{v_{H}}^{*}\right)\right)$. Denoting this difference again by $\Delta$, a proof of $x_{\left(v_{L}, x_{v_{L}}^{*}\right)}^{*}>x_{\left(v_{L}, x_{v_{H}}^{*}\right)}^{*}$ follows along the same lines as in step 2.

Step 4: $x_{\left(v_{H}, x_{v_{L}}^{*}\right)}^{*}>x_{\left(v_{L}, x_{v_{H}}^{*}\right)}^{*}$. Note that $\rho_{\left(v_{H}, x_{v_{L}}^{*}\right)}\left(\mathbf{h}_{-i, 2}\right)=\rho_{\left(v_{L}, x_{v_{H}}^{*}\right)}\left(\mathbf{h}_{-i, 2}\right)$ for all $\mathbf{h}_{-i, 2} \in$ $H_{2}$. Since the left-hand side of (32) is strictly decreasing in $x_{h_{i, 2}}^{*}, v_{H}>v_{L}$ implies that $x_{\left(v_{H}, x_{v_{L}}^{*}\right)}^{*}>x_{\left(v_{L}, x_{v_{H}}^{*}\right)}^{*}$ must hold in an interior equilibrium.

Altogether, the ranking of efforts in Proposition 7 follows by Steps 1 to 4 . Since in equilibrium all players correctly anticipate the effort of a type $\mathbf{h}_{-i, 2} \in H_{2}$ (the only difference between players emerges with respect to the beliefs $\left.\rho_{\mathbf{h}_{i, 2}}\left(\mathbf{h}_{-i, 2}\right)\right), x_{\left(v_{H}, x_{v_{H}}^{*}\right)}^{*}>x_{\left(v_{L}, x_{v_{L}}^{*}\right)}^{*}$ together with the beliefs in (29) implies (31).

In stage 2 , the highest effort is chosen by types $\left(v_{H}, x_{v_{H}}^{*}\right)$ : players who care strongly 
about winning (have a valuation $v_{H}$ ) and have faced another strong type in stage 1 . The lowest effort is chosen by low-valuation players $\left(v_{L}, x_{v_{H}}^{*}\right)$ who faced a strong type in stage 1 ; compared to types $\left(v_{L}, x_{v_{L}}^{*}\right)$, they are discouraged by their stage 1 experience. A player's expectations about the opponent's effort are still correlated with the own valuation type so that high-valuation players expect, on average, higher opponent's effort than low-valuation players, but conditional on a player's stage 1 experience this difference can disappear (compare $(31)$ ). In comparison to the efforts in stage 1, updating of beliefs can lead both to higher and to lower efforts in stage 2 .

\section{A.5 Proof of Proposition 5}

If the share $\pi_{\omega}$ of strong types is common knowledge and players only differ in their valuation $v_{i}$, an interior equilibrium $\left(\breve{x}_{v_{H}}, \breve{x}_{v_{L}}\right) \in(\underline{x}, \bar{x})^{2}$ is described by the first-order conditions ${ }^{37}$

$$
q \pi_{\omega} \frac{\breve{x}_{v_{H}}}{\left(\breve{x}_{v_{i}}+\breve{x}_{v_{H}}\right)^{2}} v_{i}+q\left(1-\pi_{\omega}\right) \frac{\breve{x}_{v_{L}}}{\left(\breve{x}_{v_{i}}+\breve{x}_{v_{L}}\right)^{2}} v_{i}-1=0 \text { for } i \in\{H, L\} .
$$

Combining the two first-order conditions yields the condition

$$
\frac{\breve{x}_{v_{L}}}{\frac{q v_{L}}{4}}\left(1-\pi_{\omega}\right)=\frac{\breve{x}_{v_{H}}}{\frac{q v_{H}}{4}} \pi_{\omega}+\left(1-2 \pi_{\omega}\right)
$$

which can be used to establish monotonicity properties of the equilibrium efforts: $\breve{x}_{v_{H}}$ is strictly increasing in $\pi_{\omega}$ and $\breve{x}_{v_{L}}$ is strictly decreasing in $\pi_{\omega} \cdot{ }^{38}$ Moreover, $\breve{x}_{v_{H}}$ and $\breve{x}_{v_{L}}$ are continuous is $\pi_{\omega}$, that is, continuous in $d$, and stage 1 equilibrium efforts $x_{v_{H}}^{*}$ and $x_{v_{L}}^{*}$ are continuous in $d$ as well. Using (7) and (8),

$$
\lim _{d \rightarrow 0} x_{v_{H}}^{*}=\frac{1}{2} \frac{q v_{H}}{4}+\frac{1}{2} \frac{q v_{H}^{2} v_{L}}{\left(v_{H}+v_{L}\right)^{2}}=\lim _{d \rightarrow 0} \breve{x}_{v_{H}}
$$

and

$$
\lim _{d \rightarrow 0} x_{v_{L}}^{*}=\frac{1}{2} \frac{q v_{L}}{4}+\frac{1}{2} \frac{q v_{H} v_{L}^{2}}{\left(v_{H}+v_{L}\right)^{2}}=\lim _{d \rightarrow 0} \breve{x}_{v_{L}}
$$

in both states $\omega \in\{\bar{\omega}, \underline{\omega}\} .^{39}$

Part (ii): Suppose that the true share of high-valuation types is $\pi_{\underline{\omega}}$. Then, $\rho_{v_{H}}\left(v_{H}\right)>$ $1 / 2>\pi_{\underline{\omega}}$ for all $d \in(0,1 / 2)$. Using (38) and the fact that $x_{v_{H}}^{*}$ is strictly increasing in

\footnotetext{
${ }^{37}$ With the minimum effort requirement of $\underline{x}>0$, the equilibrium is an interior equilibrium unless $v_{H} / v_{L}$ becomes very large. (In the latter case, low types exert equilibrium effort of $\underline{x}>0$.)

${ }^{38}$ This monotonicity result follows as in Serena (2015, Proposition 2); therefore, a proof is omitted. Intuitively, equilibrium efforts are increasing in the probability of facing a type with the same valuation.

${ }^{39}$ If $d \rightarrow 0$ then $\lim _{d \rightarrow 0} \pi_{\omega}=\lim _{d \rightarrow 0} \pi_{\omega}=\lim _{d \rightarrow 0} \rho_{v_{H}}\left(v_{H}\right)=\lim _{d \rightarrow 0} \rho_{v_{L}}\left(v_{H}\right)=1 / 2$.
} 
$\rho_{v_{H}}\left(v_{H}\right)$ (Corollary 1) and $\breve{x}_{v_{H}}$ is strictly increasing in $\pi_{\omega}$, it follows that $\breve{x}_{v_{H}}<x_{v_{H}}^{*}$ for all $d \in(0,1 / 2)$. Note that $\breve{x}_{v_{H}}\left(\pi_{\underline{\omega}}\right)$ is lowest if $d \rightarrow 1 / 2$ in which case $\breve{x}_{v_{H}}$ converges to

$$
\frac{q v_{H}^{2} v_{L}}{\left(v_{H}+v_{L}\right)^{2}}=\frac{q v_{L}}{4} \frac{\left(v_{H}+v_{H}\right)^{2}}{\left(v_{H}+v_{L}\right)^{2}}>\frac{q v_{L}}{4}
$$

hence, for all $d \in(0,1 / 2), \breve{x}_{v_{H}}\left(\pi_{\underline{\omega}}\right)$ is higher than the stage 1 effort $x_{v_{L}}^{*}$ of weak types (which is at most $q v_{L} / 4$, in case $\left.\rho_{v_{L}}\left(v_{L}\right) \rightarrow 1\right)$.

Now consider the low-valuation types. With the first-order condition for $x_{v_{L}}^{*}$,

$$
\begin{aligned}
0 & =q \rho_{v_{L}}\left(v_{H}\right) \frac{x_{v_{H}}^{*}}{\left(x_{v_{L}}^{*}+x_{v_{H}}^{*}\right)^{2}} v_{L}+q\left(1-\rho_{v_{L}}\left(v_{H}\right)\right) \frac{x_{v_{L}}^{*}}{\left(x_{v_{L}}^{*}+x_{v_{L}}^{*}\right)^{2}} v_{L}-1 \\
& <q \pi_{\underline{\omega}} \frac{x_{v_{H}}^{*}}{\left(x_{v_{L}}^{*}+x_{v_{H}}^{*}\right)^{2}} v_{L}+q\left(1-\pi_{\underline{\omega}}\right) \frac{x_{v_{L}}^{*}}{\left(x_{v_{L}}^{*}+x_{v_{L}}^{*}\right)^{2}} v_{L}-1 \\
& <q \pi_{\underline{\omega}} \frac{\breve{x}_{v_{H}}}{\left(x_{v_{L}}^{*}+\breve{x}_{v_{H}}\right)^{2}} v_{L}+q\left(1-\pi_{\underline{\omega}}\right) \frac{x_{v_{L}}^{*}}{\left(x_{v_{L}}^{*}+x_{v_{L}}^{*}\right)^{2}} v_{L}-1 .
\end{aligned}
$$

Here, the first inequality follows from $\pi_{\underline{\omega}}<\rho_{v_{L}}\left(v_{H}\right)$; the second inequality follows from $\breve{x}_{v_{H}}<x_{v_{H}}^{*}{ }^{40}$ Let $d \in(0,1 / 2)$ and suppose that $\breve{x}_{v_{L}} \leq x_{v_{L}}^{*}$. Then, the right-hand side of inequality (41) is (weakly) smaller than

$$
q \pi_{\underline{\omega}} \frac{\breve{x}_{v_{H}}}{\left(\breve{x}_{v_{L}}+\breve{x}_{v_{H}}\right)^{2}} v_{L}+q\left(1-\pi_{\underline{\omega}}\right) \frac{\breve{x}_{v_{L}}}{\left(\breve{x}_{v_{L}}+\breve{x}_{v_{L}}\right)^{2}} v_{L}-1,
$$

which is equal to zero by (36); contradiction. Therefore, we must have $\breve{x}_{v_{L}}>x_{v_{L}}^{*}$. If $d \rightarrow 1 / 2$, the probability that weak types attach to facing another weak type converges to one so that both $x_{v_{L}}^{*}$ and $\breve{x}_{v_{L}}$ converge to $q v_{L} / 4$.

Part (i): Suppose that the true share of high-valuation types is $\pi_{\bar{\omega}}$. Then, $\rho_{v_{L}}\left(v_{L}\right)>$ $1 / 2>1-\pi_{\bar{\omega}}$ for all $d \in(0,1 / 2)$. Using (39) and the fact that $x_{v_{L}}^{*}$ is strictly increasing in $\rho_{v_{L}}\left(v_{L}\right)$ (Corollary 1) and $\breve{x}_{v_{L}}$ is strictly increasing in $1-\pi_{\bar{\omega}}$, it holds that $x_{L, \mu_{1}}<x_{v_{L}}^{*}$.

Note that the proof of Proposition 5 only makes use of the rankings $\rho_{v_{L}}\left(v_{H}\right)<\pi_{\bar{\omega}}$ as well as $\rho_{v_{H}}\left(v_{H}\right)>\pi_{\underline{\omega}}$ and $\rho_{v_{L}}\left(v_{H}\right)>\pi_{\underline{\omega}}$, but does not use, for instance, that $\rho_{v_{L}}\left(v_{H}\right)=1 / 2-2 d^{2}$ and $\pi_{\underline{\omega}}=1 / 2-d$ for the assumed information structure. In other words, the result in Proposition 5 does not qualitatively depend on the players knowing the exact share of high and low players in the population. It is sufficient that the players hold common beliefs about

\footnotetext{
${ }^{40}$ The marginal probability $\partial p_{i}\left(x_{i}, x_{-i}\right) / \partial x_{i}=x_{-i} /\left(x_{i}+x_{-i}\right)^{2}$ is strictly increasing for all $x_{-i}<x_{i}$, reaches its maximum at $x_{-i}=x_{i}$, and is strictly decreasing for $x_{-i}>x_{i}$. Therefore, since $x_{v_{L}}^{*}<\breve{x}_{v_{H}}<x_{v_{H}}^{*}$, we get $x_{v_{L}}^{*} /\left(x_{v_{L}}^{*}+x_{v_{L}}^{*}\right)^{2}>\breve{x}_{v_{H}} /\left(x_{v_{L}}^{*}+\breve{x}_{v_{H}}\right)^{2}>x_{v_{H}}^{*} /\left(x_{v_{L}}^{*}+x_{v_{H}}^{*}\right)^{2}$. (The ranking $x_{v_{L}}^{*}<\breve{x}_{v_{H}}$ holds as $x_{v_{L}}^{*}<q v_{L} / 4<\breve{x}_{v_{H}}$ for all $d \in(0,1 / 2)$; see above.)
} 
the type distribution and that the beliefs about the share of high types are corrected upward in case of $\omega=\bar{\omega}$, and are corrected downward in case of $\omega=\underline{\omega}$.

In contrast, in case of $\omega=\bar{\omega}$, comparisons of $\breve{x}_{v_{H}}\left(\pi_{\bar{\omega}}\right)$ to $x_{v_{H}}^{*}$ rely on the difference between $\pi_{\bar{\omega}}$ and $\rho_{v_{H}}\left(v_{H}\right)$. It is possible (although more complex) to show that $\breve{x}_{v_{H}}\left(\pi_{\bar{\omega}}\right)>x_{v_{H}}^{*}$ for all $\left(v_{H}, v_{L}\right)$ with $v_{H}>v_{L}$ if $d$ is sufficiently small. However, $\breve{x}_{v_{H}}$ may be smaller than $x_{v_{H}}^{*}$ if $d$ is very close to $1 / 2$ and $v_{H}$ is large compared to $v_{L} \cdot{ }^{41}$

\section{A.6 Proof of Proposition 6}

Suppose all players $i$ with $v_{i}=v_{L}$ exit and all players $j$ with $v_{j}=v_{H}$ continue in stage 2. We have to show that no single player has an incentive to deviate from the candidate equilibrium behavior. In the candidate equilibrium, the players anticipate that the common beliefs among all players is that (almost) all players who remain active in stage 2 have $v_{j}=v_{H}$. Hence, players with $v_{j}=v_{H}$ choose an equilibrium effort of $x_{j, s}=q v_{H} / 4$ in all stages $s=2, \ldots, n$ and earn an expected payoff from staying active of

$$
b_{H} \equiv \frac{q v_{H}}{4} \sum_{k=2}^{k=n}(1-q)^{k-2}
$$

A player $i$ with valuation $v_{i}=v_{L}$ can also anticipate that $x_{-i, s}=q v_{H} / 4$ for all $s=2, \ldots n$ and chooses her effort $x_{i, s} \in[\underline{x}, \bar{x}]$ as the best reply. Straightforward calculus yields this best reply as $\max \left\{\tilde{x}_{L}, \underline{x}\right\}$ with

$$
\tilde{x}_{L} \equiv \frac{q}{4}\left(-v_{H}+2 \sqrt{v_{L} v_{H}}\right)
$$

at all stages $s=2, \ldots, n$. Denote the resulting continuation value for weak types by

$$
b_{L} \equiv\left(q \frac{\max \left\{\tilde{x}_{L}, \underline{x}\right\}}{\max \left\{\tilde{x}_{L}, \underline{x}\right\}+\frac{q v_{H}}{4}} v_{L}-\max \left\{\tilde{x}_{L}, \underline{x}\right\}\right) \sum_{k=2}^{k=n}(1-q)^{k-2} .
$$

Hence, it is optimal for weak types to exit and for strong types to stay if $b \in\left[b_{L}, b_{H}\right]$.

Note that the interval $\left[b_{L}, b_{H}\right]$ is non-empty. If $\tilde{x}_{L} \geq \underline{x}$ then $b_{L}$ simplifies to

$$
\frac{q}{4}\left(v_{H}-4\left(\sqrt{v_{L} v_{H}}-v_{L}\right)\right) \sum_{k=2}^{k=n}(1-q)^{k-2},
$$

which is strictly smaller than $b_{H}$ given above. If instead $\tilde{x}_{L}<\underline{x}$ then $b_{L}$ is equal to

$$
\left(q \frac{\underline{x}}{\underline{x}+\frac{q v_{H}}{4}} v_{L}-\underline{x}\right) \sum_{k=2}^{k=n}(1-q)^{k-2}<\left(q \frac{\underline{x}}{\underline{x}+\frac{q v_{H}}{4}} v_{H}-\underline{x}\right) \sum_{k=2}^{k=n}(1-q)^{k-2}<b_{H},
$$

\footnotetext{
${ }^{41}$ For instance, if the true state is $\omega=\bar{\omega}$ and $q v_{H}=390, q v_{L}=100$ and $d=0.45$, we obtain $\breve{x}_{v_{H}}=$ $94.091<94.256=x_{v_{H}}^{*}$ and $\breve{x}_{v_{L}}=8.3925<24.168=x_{v_{L}}^{*}$.
} 
where the second inequality holds whenever strong types strictly prefer an effort $q v_{H} / 4$ over an effort $\underline{x}$.

\section{A.7 Probability weighting and escalation}

A variant of the framework in Section 2.2 assumes that players have symmetric valuations of winning equal to $v$ as in the benchmark case, but that the probability of winning enters into the objective function in a non-linear fashion, in line with theories of risk-taking with weighted probabilities (see, e.g., Quiggin 1982, Yaari 1987, and Prelec 1998).

Suppose that probabilities are weighted according to a function $w:[0,1] \rightarrow[0,1]$ where $w(0)=0, w(1)=1$, and $w$ is continuous, strictly increasing and differentiable on $(0,1)$ and concave on some interval $(0, z), z>0$. If stage $s$ is reached, anticipating equilibrium efforts $\check{x}_{i, s+k}$ and equilibrium win probabilities $\check{p}_{i, s+k}$ for $k=1, \ldots, n-s$, player $i$ maximizes her expected continuation payoff

$$
w\left(q \frac{x_{i, s}}{x_{i, s}+x_{-i, s}}+\sum_{k=1}^{n-s}(1-q)^{k} q \check{p}_{i, s+k}\right) v-x_{i, s}-\sum_{k=1}^{n-s} w\left((1-q)^{k}\right) \check{x}_{i, s+k} .
$$

Straightforward calculations using symmetry (hence, $\check{p}_{i, s+k}=1 / 2$ for $k=1, \ldots, n-s$ ) yield

$$
\check{x}_{i, s}=\frac{q v}{4} w^{\prime}\left(\sum_{k=0}^{n-s}(1-q)^{k} \frac{q}{2}\right)
$$

Therefore, equilibrium efforts $\check{x}_{i, s}$ are strictly increasing in $s$ across all stages if $w$ is strictly concave on $(0, z)$ for $z=\sum_{k=0}^{n-1}(1-q)^{k} q / 2$.

As an example, let $v=450, q=1 / 3$, and $n=5$. Using the functional form

$$
w(p)=\frac{p^{\beta}}{\left(p^{\beta}+(1-p)^{\beta}\right)^{\frac{1}{\beta}}}
$$

from Baharad and Nitzan (2008) and setting $\beta=0.6$ yields equilibrium efforts $\check{x}_{i, 1}=18.327$, $\check{x}_{i, 2}=18.351, \check{x}_{i, 3}=18.677, \check{x}_{i, 4}=19.976$, and $\check{x}_{i, 5}=25.248$. 


\section{B Additional experimental results}

\section{B.1 Average efforts across rounds}

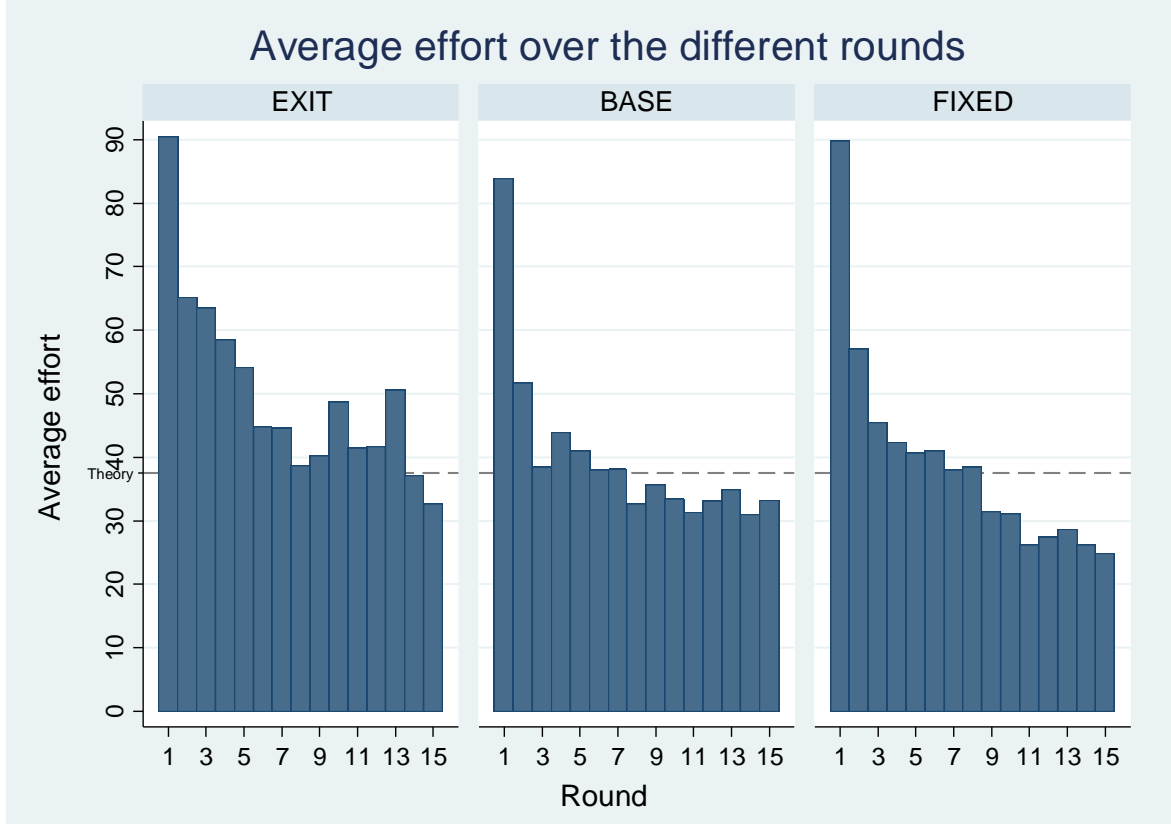

Figure B.1: Average efforts across rounds 1 to 15 (by treatment). 


\section{B.2 Average escalation by treatment}

\begin{tabular}{|c|c|c|c|c|}
\hline \multicolumn{5}{|c|}{ Dependent variable: individual effort $x_{i r s}$} \\
\hline Indep. var. & $\begin{array}{l}\text { BASE } \\
\text { xtreg } 1\end{array}$ & $\begin{array}{l}\text { FIXED } \\
\text { xtreg } 2\end{array}$ & $\begin{array}{c}\text { EXIT } \\
\text { xtreg } 3\end{array}$ & $\begin{array}{l}\text { Pooled } \\
\text { xtreg } 4\end{array}$ \\
\hline Constant & $\begin{array}{c}30.70^{* * *} \\
(5.035)\end{array}$ & $\begin{array}{c}30.23^{* * *} \\
(2.649)\end{array}$ & $\begin{array}{c}52.56^{* * *} \\
(12.281)\end{array}$ & $\begin{array}{c}36.07^{* * *} \\
(4.541)\end{array}$ \\
\hline $\mathbf{1}_{s \geq 2}$ & & & $\begin{array}{c}6.090^{* *} \\
(2.784)\end{array}$ & $\begin{array}{c}-2.985^{*} \\
(1.720)\end{array}$ \\
\hline Stage $_{s-1}$ & $\begin{array}{c}1.041^{* *} \\
(0.492)\end{array}$ & $\begin{array}{c}1.023^{* *} \\
(0.417)\end{array}$ & $\begin{array}{l}-0.401 \\
(1.218)\end{array}$ & $\begin{array}{c}1.861^{* * *} \\
(0.681)\end{array}$ \\
\hline FIXED & & & & $\begin{array}{l}-5.944 \\
(4.838)\end{array}$ \\
\hline EXIT & & & & $\begin{array}{l}7.923^{*} \\
(4.810)\end{array}$ \\
\hline FIXED $\times \mathbf{1}_{s \geq 2}$ & & & & $\begin{array}{l}2.427 \\
(2.334)\end{array}$ \\
\hline $\operatorname{EXIT} \times \mathbf{1}_{s \geq 2}$ & & & & $\begin{array}{c}9.338^{* * *} \\
(2.792)\end{array}$ \\
\hline${\text { FIXED } \times \text { Stage }_{s-1}}$ & & & & $\begin{array}{c}-0.712 \\
(0.903)\end{array}$ \\
\hline $\mathrm{EXIT} \times \operatorname{Stage}_{s-1}$ & & & & $\begin{array}{c}-2.061^{*} \\
(1.139)\end{array}$ \\
\hline Beliefs $E_{i r s}\left(x_{-i r s}\right)$ & $\begin{array}{c}0.320^{* * *} \\
(0.027)\end{array}$ & $\begin{array}{c}0.325^{* * *} \\
(0.023)\end{array}$ & $\begin{array}{c}0.286^{* * *} \\
(0.038)\end{array}$ & $\begin{array}{c}0.307^{* * *} \\
(0.016)\end{array}$ \\
\hline Beliefs $\left(E_{i r s}\left(x_{-i r s}\right)\right)^{2}$ & $\begin{array}{c}-0.0012^{* * *} \\
(0.000)\end{array}$ & $\begin{array}{c}-0.0006^{* * *} \\
(0.000)\end{array}$ & $\begin{array}{c}-0.0005^{* * *} \\
(0.000)\end{array}$ & $\begin{array}{c}-0.0007^{* * *} \\
(0.000)\end{array}$ \\
\hline$-i$ 's effort $x_{-i r 1}$ & $\begin{array}{l}-0.018 \\
(0.015)\end{array}$ & $\begin{array}{c}0.033^{* *} \\
(0.013)\end{array}$ & $\begin{array}{l}-0.021 \\
(0.015)\end{array}$ & $\begin{array}{c}0.001 \\
(0.008)\end{array}$ \\
\hline Round dummies & YES & YES & YES & YES \\
\hline Socioeconomics & YES & YES & YES & YES \\
\hline Observations & 2552 & 2784 & 1760 & 7096 \\
\hline
\end{tabular}

Note: Random-effects regressions; data from rounds 6 to 15 only; standard errors in parentheses; *** $(* *, *)$ significant at $1 \%(5 \%, 10 \%) . \mathbf{1}_{s \geq 2}=1$ if stage $\geq 2$, and $\mathbf{1}_{s \geq 2}=0$ otherwise.

Table B.1: Individual effort over stages 1 to 5. 


\section{B.3 Accuracy of beliefs over time}

\begin{tabular}{lccc}
\hline \hline \multicolumn{1}{c}{ Dependent variable: belief accuracy } & $\frac{\left|E_{i r s}\left(x_{-i r s}\right)-x_{-i r s}\right|}{E_{i r s}\left(x_{-i r s}\right)}$ & \\
& BASE & FIXED & EXIT \\
Indep. var. & xtreg 1 & xtreg 2 & xtreg 3 \\
\hline Constant & $7.981^{* * *}$ & $6.137^{* * *}$ & $7.662^{* *}$ \\
& $(1.126)$ & $(1.462)$ & $(3.368)$ \\
$\mathbf{1}_{\text {strong type }}$ & $7.297^{* * *}$ & $6.588^{* * *}$ & 1.461 \\
& $(1.656)$ & $(2.181)$ & $(3.803)$ \\
Stage contest \\
& $-0.338^{* * *}$ & $-0.320^{* * *}$ & $-0.496^{* * *}$ \\
& $(0.105)$ & $(0.120)$ & $(0.192)$ \\
$\mathbf{1}_{\text {strong type }} \times$ Stage contest $_{r \times s}$ & $-0.589^{* * *}$ & -0.240 & -0.353 \\
& $(0.162)$ & $(0.197)$ & $(0.306)$ \\
$($ Stage contest \\
\end{tabular}

Note: Random-effects regressions; data from rounds 1 to 15 ; standard errors in parentheses; *** $\left(* * *{ }^{*}\right)$ significant at $1 \%(5 \%, 10 \%)$. Dependent variable is the absolute value of the difference of stated beliefs and actual opponent's effort, divided by stated beliefs. $\mathbf{1}_{\text {strong type }}=1$ if subject $i$ 's average effort in rounds 1 to 5 higher than average effort of all subjects in rounds 1 to 5 of the respective treatment, and $\mathbf{1}_{\text {strong type }}=0$ otherwise. "Stage contest $r \times s$ " is the number of the stage contest played (from 1 to 44 ).

Table B.2: Updating of beliefs: Belief accuracy over the stage contests played. 


\section{B.4 Stated beliefs across the stages}

\begin{tabular}{|c|c|c|c|c|}
\hline \multicolumn{5}{|c|}{ Dependent variable: beliefs $E_{i r s}\left(x_{-i r s}\right)$} \\
\hline Indep. var. & $\begin{array}{l}\text { BASE } \\
\text { xtreg } 1\end{array}$ & $\begin{array}{l}\text { FIXED } \\
\text { xtreg } 2\end{array}$ & $\begin{array}{c}\text { EXIT } \\
\text { xtreg } 3\end{array}$ & $\begin{array}{c}\text { BASE } \\
\text { xtreg } 4\end{array}$ \\
\hline Constant & $\begin{array}{c}27.69^{* * *} \\
(3.927)\end{array}$ & $\begin{array}{c}27.63^{* * *} \\
(2.936)\end{array}$ & $\begin{array}{c}26.15^{*} \\
(14.431)\end{array}$ & $\begin{array}{c}36.52^{* * *} \\
(7.414)\end{array}$ \\
\hline $\mathbf{1}_{\text {strong type }}$ & $\begin{array}{c}13.55^{* * *} \\
(4.532)\end{array}$ & $\begin{array}{c}18.57^{* * *} \\
(3.338)\end{array}$ & $\begin{array}{c}13.45 \\
(12.780)\end{array}$ & $\begin{array}{c}34.75^{* * *} \\
(8.574)\end{array}$ \\
\hline Stage $_{s-1}$ & $\begin{array}{c}-0.595 \\
(0.667)\end{array}$ & $\begin{array}{c}-0.577 \\
(0.598)\end{array}$ & & $\begin{array}{c}-8.528^{* * *} \\
(1.883)\end{array}$ \\
\hline $\mathbf{1}_{\text {strong type }} \times$ Stage $_{s-1}$ & $\begin{array}{c}-2.085^{* *} \\
(0.934)\end{array}$ & $\begin{array}{c}-0.478 \\
(0.880)\end{array}$ & & $\begin{array}{c}-3.558 \\
(2.660)\end{array}$ \\
\hline $\mathbf{1}_{s \geq 2}$ & & & $\begin{array}{l}1.488 \\
(2.971)\end{array}$ & \\
\hline $\mathbf{1}_{\text {strong type }} \times \mathbf{1}_{s \geq 2}$ & & & $\begin{array}{c}-3.119 \\
(4.692)\end{array}$ & \\
\hline$-i$ 's effort $x_{-i r 1}$ & $\begin{array}{c}0.038 * * \\
(0.016)\end{array}$ & $\begin{array}{c}0.152^{* * *} \\
(0.015)\end{array}$ & $\begin{array}{c}0.087^{* * *} \\
(0.022)\end{array}$ & $\begin{array}{c}0.073^{* * *} \\
(0.025)\end{array}$ \\
\hline Round dummies & YES & YES & YES & YES \\
\hline Socioeconomics & YES & YES & YES & YES \\
\hline Observations & 2552 & 2784 & 1760 & 1320 \\
\hline
\end{tabular}

Note: Random-effects regressions; estimations (1)-(3) based data from rounds 6 to 15; estimation (4) based data from rounds 1 to 5 ; standard errors in parentheses; ***(**,*) significant at $1 \%(5 \%, 10 \%)$. $\mathbf{1}_{\text {strong type }}=1$ if subject $i$ 's average effort in rounds 1 to 5 higher than average effort of all subjects in rounds 1 to 5 of the respective treatment, and $\mathbf{1}_{\text {strong type }}=0$ otherwise. $\mathbf{1}_{s \geq 2}=1$ if stage $\geq 2$, and $\mathbf{1}_{s \geq 2}=0$ otherwise.

Table B.3: Updating of beliefs over stages 1 to 5: Strong vs. weak types. 


\section{References}

[1] Baharad, Eyal, and Shmuel Nitzan, 2008, Contest efforts in light of behavioral considerations, Economic Journal, 118(533), 2047-2059.

[2] Baye, Michael R., and Heidrun C. Hoppe, 2003, The strategic equivalence of rentseeking, innovation, and patent-race games, Games and Economic Behavior, 44(2), 217226.

[3] Baye, Michael R., Dan Kovenock, and Casper G. de Vries, 1993, Rigging the lobbying process: An application of the all-pay auction, American Economic Review, 83(1), 289294.

[4] Ben-Bassat, Avi, 2011, Conflicts, interest groups, and politics in structural reforms, Journal of Law \& Economics, 54(4), 937-952.

[5] Brookins, Philip and Dmitry Ryvkin, 2014, An experimental study of bidding in contests of incomplete information, Experimental Economics, 17(2), 245-261.

[6] Che, Yeon-Koo, and Ian Gale, 2003, Optimal design of research contests, American Economic Review, 93(3), 646-671.

[7] Clark, Derek J., and Christian Riis, 1998, Contest success functions: An extension, Economic Theory, 11(1), 201-204.

[8] Dechenaux, Emmanuel, Dan Kovenock, and Roman Sheremeta, 2015, A survey of experimental research on contests, all-pay auctions and tournaments, Experimental Economics, 18(4), 609-669.

[9] Deck, Cary, and Roman M. Sheremeta, 2012, Fight or flight? Defending against sequential attacks in the game of siege, Journal of Conflict Resolution, 56(6), 1069-1088.

[10] Deck, Cary, and Roman M. Sheremeta, 2015, Tug-of-war in the laboratory, available at SSRN: https://ssrn.com/abstract $=2658795$.

[11] Einy, E., O. Haimankoa, D. Moreno, A. Sela, and B. Shitovitz, 2015, Equilibrium existence in Tullock contests with incomplete information, Journal of Mathematical Economics, 61, 241-245.

[12] Ellingsen, Tore, 1991, Strategic buyers and the social cost of monopoly, American Economic Review, 81(3), 648-657. 
[13] Fey, Mark, 2008, Rent-seeking contests with incomplete information, Public Choice,135(3-4), 225-236.

[14] Fu, Qiang, Oliver Gürtler, and Johannes Münster, 2013, Communication and commitment in contests, Journal of Economic Behavior \& Organization, 95, 1-19.

[15] Fullerton, Richard L., and R. Preston McAfee, 1999, Auctioning entry into tournaments, Journal of Political Economy, 107(3), 573-605.

[16] Gelder, Alan, and Dan Kovenock, 2017, Dynamic behavior and player types in majoritarian multi-battle contests, Games and Economic Behavior, 104, 444-455.

[17] Greiner, Ben, 2004, An online recruitment system for economic experiments. In: K. Kremer and V. Macho (eds.), Forschung und wissenschaftliches Rechnen 2003, GWDG Bericht 63, Göttingen, Ges. für Wiss. Datenverarbeitung, 79-93.

[18] Harris, Christopher, and John Vickers, 1987, Racing with uncertainty, Review of Economic Studies, 54(1), 1-21.

[19] Herbst, Luisa, 2016, Who pays to win again? The joy of winning in contest experiments, Working Paper of the Max Planck Institute for Tax Law and Public Finance No. 2016-6.

[20] Herbst, Luisa, Kai A. Konrad, and Florian Morath, 2015. Endogenous group formation in experimental contests, European Economic Review, 74, 163-189.

[21] Hirshleifer, Jack, and John G. Riley, 1992, The Analytics of Uncertainty and Information, Cambridge: Cambridge University Press.

[22] Jia, Hao, 2008, A stochastic derivation of the ratio form of contest success functions, Public Choice, 135(3-4), 125-130.

[23] Kawada, Christie L.K., Peter M. Gollwitzer, Gabriele Oettingen, and John A. Bargh, 2004, The projection of implicit and explicit goals, Journal of Personality and Social Psychology, 86(4), 545-559.

[24] Kerschbamer, Rudolf, Matthias Sutter, and Uwe Dulleck, 2017, How social preferences shape incentives in (experimental) markets for credence goods, Economic Journal, 127(600), 393-416.

[25] Klumpp, Tilman and Mattias K. Polborn, 2006, Primaries and the New Hampshire effect, Journal of Public Economics, 90(6-7), 1073-1114. 
[26] Konrad, Kai A., 2009, Strategy and Dynamics in Contests, Oxford: Oxford University Press.

[27] Konrad, Kai A., and Dan Kovenock, 2015, Interest groups, influence activities and politicians with imperfect commitment, unpublished manuscript, presented at the SAET meeting 2015 in Paris.

[28] Krispenz, Ann, Oliver Dickhäuser, and Marc-André Reinhard, 2016, Assessing task difficulty for other people: When deeper evaluation means "it's more about me!", Social Psychology of Education, 19(4), 865-877.

[29] Lee, Tom, and Louis L. Wilde, 1980, Market structure and innovation: A reformulation, Quarterly Journal of Economics, 94(2), 429-436.

[30] Loury, Glenn C., 1979, Market structure and innovation, Quarterly Journal of Economics, 93(3), 395-410.

[31] Lugovskyy, Volodymyr, Daniela Puzzello, and Steven Tucker, 2010, An experimental investigation of overdissipation in the all pay auction, European Economic Review, 54(8), 974-997.

[32] Mago, Shakun D., Roman M. Sheremeta, and Andrew Yates, 2013, Best-of-three contest experiments: Strategic versus psychological momentum, International Journal of Industrial Organization, 31(3), 287-296.

[33] Malueg, David A., and Andrew J. Yates, 2004, Rent seeking with private values, Public Choice, 119(1-2), 161-178.

[34] Marks, Gary, and Norman Miller, 1987, Ten years of research on the false-consensus effect: An empirical and theoretical review, Psychological Bulletin, 102(1), 72-90.

[35] Meirowitz, Adam, 2008, Electoral contests, incumbency advantages, and campaign finance, Journal of Politics, 70(3), 681-699.

[36] Münster, Johannes, 2009, Repeated contests with asymmetric information, Journal of Public Economic Theory, 11(1), 89-118.

[37] Nickerson, Raymond S., 1998, Confirmation bias: A ubiquitous phenomenon in many guises, Review of General Psychology, 2(2), 175-220.

[38] Prelec, Drazen, 1998, The probability weighting function, Econometrica, 66(3), 497-527. 
[39] Quiggin, John, 1982, A theory of anticipated utility, Journal of Economic Behavior and Organization, 3(4), 324-343.

[40] Ryvkin, Dmitry, 2010, Contests with private costs beyond two players, European Journal of Political Economy, 26(4), 558-567.

[41] Serena, Marco, 2016, Harnessing beliefs to stimulate efforts, available at SSRN: https://ssrn.com/abstract $=2686543$.

[42] Sheremeta, Roman M., 2013, Overbidding and heterogeneous behavior in contest experiments, Journal of Economic Surveys, 27(3), 491-514.

[43] Skaperdas, Stergios, 1996, Contest success functions, Economic Theory, 7(2), 283-290.

[44] Tullock, Gordon, 1980, Efficient rent-seeking. In: J. Buchanan et al. (eds.), Toward a Theory of the Rent-Seeking Society, College Station: Texas A\&M University Press.

[45] Wärneryd, Karl, 2012, Multi-player contests with asymmetric information, Economic Theory, 51(2), 277-287.

[46] Wasser, Cédric, 2013a, A note on Bayesian Nash equilibria in imperfectly discriminating contests, Mathematical Social Sciences, 66(2), 180-182.

[47] Wasser, Cédric, 2013b, Incomplete information in rent-seeking contests, Economic Theory, 53(1), 239-268.

[48] Yaari, Menahem E., 1987, The dual theory of choice under risk, Econometrica, 55(1), 95-115. 